\title{
Geometry of the Large Magellanic Cloud Using Multi- wavelength Photometry of Classical Cepheids
}

\author{
Sukanta Deb ${ }^{1,2 \star}$, Chow-Choong Ngeow ${ }^{3}$, Shashi M. Kanbur ${ }^{4}$, Harinder P. Singh ${ }^{5}$, \\ Daniel Wysocki ${ }^{6}$, Subhash Kumar ${ }^{7}$ \\ ${ }^{1}$ Department of Physics, Cotton University, Panbazar, Guwahati 781001, Assam, India \\ ${ }^{2}$ Space and Astronomy Research Center, Cotton University, Panbazar, Guwahati 781001, Assam, India \\ ${ }^{3}$ Graduate Institute of Astronomy, National Central University, Jhongli 32001, Taiwan \\ ${ }^{4}$ Department of Physics, State University of New York at Oswego, Oswego, NY 13126, USA \\ ${ }^{5}$ Department of Physics \&S Astrophysics, University of Delhi, Delhi 110007, India \\ ${ }^{6}$ Center for Computational Relativity and Gravitation, Rochester Institute of Technology, Rochester, NY 14623, USA \\ ${ }^{7}$ Department of Physics, Acharya Narendra Dev College, Govindpuri, Kalkaji, New Delhi 110019, India
}

Received on ; Accepted on

\begin{abstract}
We determine the geometrical and viewing angle parameters of the Large Magellanic Cloud (LMC) using the Leavitt law based on a sample of more than 3500 common classical Cepheids (FU and FO) in optical $(V, I)$, near-infrared $\left(J H K_{s}\right)$ and midinfrared ([3.6] $\mu \mathrm{m}$ and [4.5] $\mu \mathrm{m})$ photometric bands. Statistical reddening and distance modulus free from the effect of reddening to each of the individual Cepheids are obtained using the simultaneous multi-band fit to the apparent distance moduli from the analysis of the resulting Leavitt laws in these seven photometric bands. A reddening map of the LMC obtained from the analysis shows good agreement with the other maps available in the literature. Extinction free distance measurements along with the information of the equatorial coordinates $(\alpha, \delta)$ for individual stars are used to obtain the corresponding Cartesian coordinates with respect to the plane of the sky. By fitting a plane solution of the form $z=f(x, y)$ to the observed three dimensional distribution, the following viewing angle parameters of the LMC are obtained: inclination angle $i=25^{\circ} .110 \pm 0^{\circ} .365$, position angle of line of nodes $\theta_{\text {lon }}=154^{\circ} .702 \pm 1^{\circ} .378$. On the other hand, modelling the observed three dimensional distribution of the Cepheids as a triaxial ellipsoid, the following values of the geometrical axes ratios of the LMC are obtained: $1.000 \pm 0.003: 1.151 \pm 0.003: 1.890 \pm 0.014$ with the viewing angle parameters: inclination angle of $i=11^{\circ} .920 \pm 0^{\circ} .315$ with respect to the longest axis from the line of sight and position angle of line of nodes $\theta_{\text {lon }}=128^{\circ} .871 \pm 0^{\circ} .569$. The position angles are measured eastwards from north.
\end{abstract}

Key words: stars: variables: Cepheids - stars: statistics - stars: distances -(galaxies:) Magellanic Clouds - methods: data analysis - methods: statistical

\section{INTRODUCTION}

The LMC is classified as a barred spiral galaxy of type $\mathrm{SB}(\mathrm{s}) \mathrm{m}$, the prototype of a class of Magellanic spirals (de Vaucouleurs \& Freeman 1972; de Vaucouleurs et al. 1991). The galaxy is characterised by a prominent offset stellar bar located near its center with the dominant spiral arm to the north with two "embryonic" arms situated to the south. The galaxy is situated at a distance of $49.97 \pm 0.19$ (stat.) \pm 1.11 (sys.) kpc (Pietrzyński et al. 2013). Because of its proximity to the Milky Way, the LMC offers

* E-mail: sukanta.deb@cottonuniversity.ac.in an excellent laboratory to address many important astrophysical issues such as star formation, morphological structure, dust distribution using various tracers, microlensing observed towards the LMC, gravitational and hydrodynamical interaction as well as structure formation and evolution of galaxies. The accurate distance determination to the LMC plays a pivotal role in calibrating the extragalactic distance scale. Over the past two decades, considerable progress has been made in reducing the systematic uncertainty in the LMC distance determination in order to increase the accuracy of the value of the Hubble constant $H_{0}$.

Cepheids have played a vital role in the extragalactic distance determinations in astrophysics since the discovery 
of the period-luminosity (PL) relations called the Leavitt law (Leavitt 1908; Leavitt \& Pickering 1912). Since Cepheids are young stars, they are found in abundance in a spiral galaxy such as the LMC (Freedman et al. 2001). Because of negligible values of interstellar extinction (reduced by a factor of $\sim 20$ compared to optical wavelengths) and reduced sensitivity to metallicity, longer wavelength Cepheid data have been utilized in a large number of studies to calibrate the LMC Cepheid PL relations for distance determinations: outlined in Ngeow et al. (2009b), Freedman et al. (2011), Bhardwaj et al. (2016a), Bhardwaj et al. (2016b) and Bhardwaj et al. (2017). Using the mid-infrared observations of Spitzer, a large number of classical Cepheid data were collected by SAGE (Surveying the Agents of a Galaxy's Evolution) project (Meixner et al. 2006). Based on these single epoch observations, Ngeow \& Kanbur (2008); Freedman et al. (2008), among others, derived mid-infrared PL relations for LMC fundamental mode Cepheids at four wavelength bands.

The use of multi-wavelength PL relations of Cepheids in the extra-galactic distance-scale studies has a number of advantages: (i) Distance determinations are less affected due to the differences in chemical composition from one galaxy to the other/among different regions of the same galaxy/among different photometric bands and hence consistent values of distances are possible, (ii) Dependence of zero points of the Cepheid PL relations on metallicity can be minimised once corrected for reddening using multi-wavelength PL relations and hence the systematic error in distance determination can be reduced significantly, (iii) Independent determinations of true distance modulus and reddening (Freedman \& Madore 1990; Freedman et al. 2001). However, the extinction corrections due to interstellar reddening rely on the assumption of a universal behaviour of Cepheids at different wavelengths and a universality of the Galactic extinction law (Freedman et al. 2001). The independent reddening values obtained from the multi-wavelength PL relations are crucial to construct reddening map of the host galaxy which is important to study the dust distribution among different regions of the host galaxy. Furthermore, the use of multiwavelength photometry using more number of bands not only increases the figure of merit ratio but also takes into account complex interplay among magnitudes in different photometric bands. This helps in disentangling part of magnitude variations due to distance and other factors like age, metallicity, reddening, etc., (Nikolaev et al. 2004).

The road map of the present investigation using the multi-wavelength archival data of common LMC classical Cepheids in seven photometric bands, viz., $V, I, J, H, K,[3.6] \mu m$ and [4.5] $\mu m$ consists of the following : i) new improved PL relations of classical Cepheids using the Nikolaev et al. (2004) method, ii) independent determinations of accurate values of reddening and reddeningcorrected distances to the individual classical Cepheids with respect to the mean reddening and distance of the LMC from the simultaneous solutions of multi-band data, iii) highly precise and accurate reddening map as well as geometrical and viewing angle parameters of the LMC, iv) Separation of bar and disk of the LMC as well as determinations of the viewing angle parameters for these two distinct structures using the projected Cartesian coordinates. The Cartesian coordinates and the distance values obtained from the multi- wavelength analysis also facilitate the comparison of the line of sight distances among different regions of the LMC disk based on a statistical test.

One of the important reasons for using the data only for the mid-infrared [3.6] $\mu \mathrm{m}$ and [4.5] $\mu \mathrm{m}$ photometric bands from the existing four mid-infrared bands is that the magnitude values are available for a statistically large sample of classical Cepheids in these two photometric bands from the SAGE catalog corresponding to the common stars with $V, I, J, H, K s$ observations from Inno et al. (2016). The catalog compiled by Inno et al. (2016) contains the largest data set for classical Cepheids in the near infrared based on the OGLE-IV catalog (Soszyński et al. 2015). The near-infrared mean magnitudes were compiled in Inno et al. (2016) using the data from the VMC survey, LMC NIR Synoptic survey, IRSF sample, 2MASS catalog as well as from Persson et al. (2004). Mostly, the near-infrared mean magnitudes not available in those databases were determined in the catalog based on the template fitting technique developed by Inno et al. (2015), whereas the determinations of the OGLE-IV optical mean magnitudes were based on the seventh order Fourierseries fit. Further details of the LMC Cepheid catalog compilation can be found in Inno et al. (2016).

The present sample of classical Cepheids is restricted to those stars which have the complementary data in all seven wavelength photometric bands. One of the first studies dealing with the determination of the geometry of the disk of the LMC using a large sample of classical Cepheids (> 2000) with an improved areal coverage was that of Nikolaev et al. (2004). The study of Nikolaev et al. (2004) utilised multiwavelength photometric data based on the MACHO (MAssive Compact Halo Objects) $V$ and $R$-band light curves with complete phase coverage and single epoch photometry from 2MASS (Two Micron All Sky Survey) $J H K_{s}$ observations. The method developed by Nikolaev et al. (2004) is a very robust and efficient one in the determination of distance and reddening to each of the individual Cepheids with respect to the mean distance and reddening of the LMC using the simultaneous multi-band photometric data.

During the last few decades, a large number of studies have been devoted to the determination of the structure of the LMC using classical Cepheids. Most of the studies were based either on the PL or PW (period-Wesenheit) relations by employing an iterative $k \sigma$ clipping algorithm to clean these relations from the presence of outliers. Nonetheless, the sigma clipping algorithms to perform the rejection of outliers in these studies were arbitrary. For example, Inno et al. (2016) used $6 \sigma$ clipping before the PW relations were fitted. On the other hand, $3 \sigma$ clipping was used by Jacyszyn-Dobrzeniecka et al. (2016) to remove the outliers from the resulting PW relations. One of the important drawbacks to using such an outlier removal algorithm is that it is based on the assumption that the errors are distributed normally. But it has been demonstrated by Nikolaev et al. (2004) that this assumption is invalid for distances and reddenings.

In the present study, we apply the method developed by Nikolaev et al. (2004) to the simultaneously available multi-wavelength data for common classical Cepheids in seven photometric bands in order to independently determine the distance and reddening of individual Cepheids with respect to the mean distance and reddening of the 
LMC. Section 2 deals with the data and sample selection procedure. The present sample for classical Cepheids are based on the data available in seven photometric bands, viz., $V, I, J, H, K,[3.6] \mu m$ and [4.5] $\mu m$ chosen from the literature. The methodology adopted in the present study to fit the $P L$ relations in the seven photometric bands is described in Section 3. The application of this method to obtain the $P L$ relations with and without corrections of distance as well as reddening values in seven photometric bands is described in Section 4. Distance and reddening values for each of the individual Cepheids with respect to the mean distance and reddening of the LMC obtained from the simultaneous solutions of apparent distance moduli are also discussed in that Section. A reddening map of the LMC is presented in Section 5. The map is constructed from the reddening values of individual Cepheids along with the information on their Cartesian coordinates derived from the values of distance and equatorial $(\alpha, \delta)$ - coordinates as provided in the cata$\log$ of Inno et al. (2016). Determination of the geometrical and viewing angle parameters of the LMC obtained from the three dimensional $(x, y, z)$ distributions for all the Cepheids is discussed in Section 6. In Section 7 we separate the bar and disk Cepheids based on inequalities defined in the $X Y$ plane and study their orientations and offsets using the plane fitting procedure. Based on statistical tests, we also find the closest and farthest parts of the LMC. The summary and conclusions of this study are presented in Section 8.

\section{DATA AND SAMPLE SELECTION}

We use the 3920 classical Cepheid mean magnitudes available in the $V, I, J, H, K s$ photometric bands along with the information of periods $(P)$ and equatorial coordinates $(\alpha, \delta)$ collected by Inno et al. (2016). The mid-infrared data for magnitudes obtained in the photometric bands [3.6] $\mu \mathrm{m}$ and [4.5] $\mu m$ taken from the SAGE catalog (Meixner et al. 2006) were cross matched with Inno et al. (2016) sample using the X-Match service of VizieR ${ }^{1}$ based on the $(\alpha, \delta)$ values within the search radius of $1^{\prime \prime}$. The query returned 3639 stars which contains single epoch observations in the [3.6] $\mu \mathrm{m}$ and [4.5] $\mu \mathrm{m}$ photometric bands. Out of 3639 stars, 3614 stars have magnitudes in both the two photometric bands. Since the amplitudes of Cepheids in the mid-infrared bands are very small, the single epoch measurements are taken as the approximate values of mean magnitudes corresponding to these bands. The mid-infrared bands are located in the Rayleigh-Jeans tail of the blackbody spectrum. The effect of temperature changes are minimum in this part of the spectrum which result into smaller amplitudes as well as highly symmetrical nature of the light curves of Cepheids (Ngeow \& Kanbur 2008; Freedman et al. 2008; Ngeow et al. 2010; Scowcroft et al. 2016). It has been demonstrated by Ngeow \& Kanbur (2008) that the PL relations obtained from the mid-infrared bands agree quite well with each other when these relations are obtained with or without random phase corrections due to the small values of amplitudes in these photometric bands. We have chosen only those common stars which have mean magnitudes in all the seven pho-

1 http://vizier.u-strasbg.fr/viz-bin/VizieR tometric bands. Their numbers turn out to be 3614 out of which there are 2112 fundamental mode (FU) Cepheids and 1502 first overtone (FO) Cepheids.

We would like to point out important differences between the study done by Inno et al. (2016) and the present study. In the study of Inno et al. (2016), the PW relations for 3920 Cepheids at optical and Near infrared were utilised for relative distance determinations of individual Cepheids. Relative distances were then converted into individual absolute distances using the mean distance modulus to the LMC $\mu_{0, L M C}=18.493$ mag taken from Pietrzyński et al. (2013). The viewing angle parameters were calculated by fitting a plane of the form $z=f(x, y)$ to the Cartesian $(x, y, z)$ distributions obtained from the transformation equations connecting $(\alpha, \delta, D) \rightarrow(x, y, z)$. Because $\mathrm{PW}$ relations were used, the measured distances are free from interstellar extinction. Based on a common sample of $\sim 2600$ Cepheids with the values of apparent distance moduli available in six photometric bands $V, I, J, H, K_{S}$ and $w 1$, a reddening law was fitted simultaneously to the apparent distance moduli as a function of inverse wavelength to determine the true distance modulus and reddening for individual Cepheids. A reddening map was also provided by Inno et al. (2016) based on the reddening values of these 2600 Cepheids. But the values of the true distance moduli obtained from the reddening law fit were not used by Inno et al. (2016) to measure the viewing angle parameters of the LMC. Also, the calculation of geometrical parameters such as the axes ratios were not attempted in that study.

On the other hand, we do not use the PW relations to investigate the three dimensional structure of the LMC. The geometrical and viewing angle parameters of the LMC are obtained using the multi-wavelength PL relations for a common sample of 3614 Cepheids in seven photometric bands. The relative values of distance modulus as well as reddening are obtained from the simultaneous fitting of the seven relative apparent distance moduli using a reddening law as a function of inverse wavelength (Cardelli et al. 1989). We prove that when these PL relations are corrected using the same set of obtained values of reddening and distance for individual Cepheids from the simultaneous multi-wavelength fitting, a remarkable reduction in the dispersions of the all $\mathrm{PL}$ relations is achieved. The distance moduli and reddening offset values of individual Cepheids are converted into their absolute values using the LMC mean values of these parameters taken from the literature. Apart from finding the viewing angle parameters of the LMC, determination of its geometrical parameters such as axes ratios has also been carried out in the present study.

\section{METHODOLOGY}

We follow Nikolaev et al. (2004) to derive the statistical reddening and distance to each of the present sample of individual Cepheids. Availability of the values of mean magnitudes of the Cepheids in multi-wavelength bands allows us to disentangle the effect of reddening and distance which produce a scatter in the observed PL relations of the Cepheids. We know that the true distance modulus $\left(\mu_{0}\right)$ is related to the observed distance modulus $\left(\mu_{\lambda}\right)$ in a particular photometric 
band $(\lambda)$ as follows:

$$
\begin{aligned}
\mu_{0} & =\mu_{\lambda}-A_{\lambda} \\
\Rightarrow \mu_{0} & =\left(\bar{m}_{\lambda}-M_{\lambda}\right)-R_{\lambda} E(B-V) \\
\Rightarrow \bar{m}_{\lambda} & =M_{\lambda}+\mu_{0}+R_{\lambda} E(B-V),
\end{aligned}
$$

where $\bar{m}_{\lambda}$ and $M_{\lambda}$ denote the apparent mean magnitude and absolute magnitude of a Cepheid, respectively in a particular photometric band $(\lambda) . R_{\lambda}=\frac{A_{\lambda}}{E(B-V)}$ denotes the ratio of total to selective absorption in photometric band $\lambda$. It is obtained from a reddening law and is held fixed. Cepheids obey a PL relation given by the Leavitt law:

$M_{\lambda}=\alpha_{\lambda} \log P+\beta_{\lambda}+\epsilon_{\lambda}\left(M, T_{\text {eff }}, Z, \ldots\right)$,

where $\alpha_{\lambda}$ and $\beta_{\lambda}$ denote the PL coefficients. $\epsilon_{\lambda}$ denotes the unknown correction term related to the stellar parameters such as mass $(M)$, effective temperature $\left(T_{\text {eff }}\right)$, metallicity $(Z)$ or other parameters due to the non-linearity of the PL relation in a particular photometric band $(\lambda)$. Substituting the value of $M_{\lambda}$ into equation (1), we get

$\bar{m}_{\lambda}=\alpha_{\lambda} \log P+\beta_{\lambda}+\mu_{0}+R_{\lambda} E(B-V)+\epsilon_{\lambda}\left(M, T_{\text {eff }}, Z, \ldots\right)$

Since the general form of the correction term $\epsilon_{\lambda}$ is unknown, we assume that it is normally distributed with zero mean and variance $\sigma_{0, \lambda}^{2}$, i.e. $\epsilon_{\lambda} \sim N\left(0, \sigma_{0, \lambda}^{2}\right)$. The unknown function $\epsilon_{\lambda}$ is responsible for the physics governing the dispersion of the observed PL relation in a particular photometric band $(\lambda)$. This can be accommodated into the zero point $\left(\beta_{\lambda}\right)$ resulting into $\beta_{\lambda}^{\prime}$. Writing the above equation for each individual Cepheid in a particular photometric band $(\lambda)$, we have

$\bar{m}_{\lambda, i}=\alpha_{\lambda} \log P_{i}+\beta_{\lambda}+\mu_{0, i}+R_{\lambda} E(B-V)_{i}+\epsilon_{\lambda}\left(M, T_{\mathrm{eff}}, Z, \ldots\right)$

Let $\overline{E(B-V)}$ LMC and $\bar{\mu}_{L M C}$ denote the average values of the reddening and distance modulus of the LMC. Let us denote

$$
\begin{aligned}
\Delta E(B-V)_{i} & =E(B-V)_{i}-\overline{E(B-V)}_{\mathrm{LMC}}, \\
\Delta \mu_{0, i} & =\mu_{0, i}-\bar{\mu}_{L M C} .
\end{aligned}
$$

Therefore, we have the following equation for $\bar{m}_{\lambda}$ :

$\bar{m}_{\lambda, i}=\alpha_{\lambda} \log P_{i}+\beta_{\lambda}^{\prime}+\Delta \mu_{0, i}+R_{\lambda} \Delta E(B-V)_{i}$.

Here $\beta_{\lambda}^{\prime}$ denotes the now zero point which incorporates $\epsilon_{\lambda}$ and the mean values $\bar{\mu}_{L M C}$ and $\overline{E(B-V)_{\text {LMC }}}$. The values of $\bar{m}_{\lambda}$ corresponding to the photometric band $\lambda=(V, I, J, H, K s,[3.6],[4.5])$ and period $(P)$ for each star are given. The central wavelengths of the seven photometric bands are chosen as $\lambda_{V, I, J, H, K s,[3.6,4.5]}=\{0.555,0.790,1.235,1.662,2.159,3.550$,

4.493\} $\mu \mathrm{m}$ (Bonanos et al. 2010). Using the Cardelli et al. (1989) reddening law, the corresponding reddening values are found to be $R_{\lambda}=\{3.23,2.05,0.94,0.58,0.38,0.17,0.12\}$ with the ratios of interstellar extinction as $\frac{A_{\lambda}}{A_{V}}=$ $\{1.0,0.64,0.29,0.18,0.12,0.05,0.04\}$. The above system of equations given by equation (3) can be solved using a general linear least-squares minimisation method to find the PL coefficients $\left(\alpha_{\lambda}, \beta_{\lambda}^{\prime}\right)$ for each particular photometric band. This information obtained from the multi-wavelength band PL relations can be used to obtain the values of reddening and distance for an individual Cepheid. Equation (3) can be solved in two iterations. In the first iteration, the coefficients $\left(\alpha_{\lambda}, \beta_{\lambda}^{\prime}\right)$ are obtained by approximating the observed mean apparent magnitude $\bar{m}_{\lambda}$ as:

$\bar{m}_{\lambda, i} \approx \alpha_{\lambda} \log P_{i}+\beta_{\lambda}^{\prime}$.

The values of these coefficients $\left(\alpha_{\lambda}, \beta_{\lambda}^{\prime}\right)$ obtained in this iteration obtained from the multi-wavelength photometric band $(\lambda)$ are then used to calculate the reddening and distance to each individual Cepheid. The least-squares fitting here is done using unweighted method with the weights $w_{i}=1.0$ and does not involve the magnitude uncertainty for each of the Cepheids in a particular photometric band. In the second iteration, equation (3) is solved using the weighted least-squares minimisation method taking into account the distance and reddening values obtained from the first iteration with the weights $w_{i}=\sigma_{i}^{-2}$, where

$\sigma_{i}^{2}=\sigma_{0}^{2}+\sigma_{\text {phot }}^{2}+\sigma_{\alpha}^{2} \log ^{2} P_{i}+\sigma_{\beta}^{2}$.

Here $\sigma_{0}, \sigma_{\text {phot }}, \sigma_{\alpha} \log P_{i}$ and $\sigma_{\beta}$ denote the intrinsic error, photometric errors, the errors in the slopes and intercepts of the PL relations obtained in iteration 1, respectively. Following Nikolaev et al. (2004), we assume $\sigma_{0}=0.05 \mathrm{mag}$, irrespective of the photometric band. The distance and reddening values are obtained in the following way after $\left(\alpha_{\lambda}, \beta_{\lambda}^{\prime}\right)$ are determined from the first iteration:

$$
\begin{aligned}
\Delta \mu_{\lambda, i} & =\bar{m}_{\lambda, i}-\left(\alpha_{\lambda} \log P_{i}+\beta_{\lambda}^{\prime}\right) \\
\Rightarrow \Delta \mu_{\lambda, i} & =\Delta \mu_{0, i}+R_{\lambda} \Delta E(B-V)_{i} .
\end{aligned}
$$

The true distance modulus $\Delta \mu_{0, i}$ and reddening $\Delta E(B-V)_{i}$ for each star with respect to the mean values of the LMC can be obtained from iteration 1 by plotting the apparent relative distance moduli $\Delta \mu_{\lambda, i}$ as a function of inverse wavelength $\lambda^{-1}$ for seven photometric bands with the assumption that wavelength dependence of these relative distance moduli is due to extinction. The amount of extinction at a given wavelength is inversely proportional to the wavelength (Freedman 1988). All the data are then fitted simultaneously with an interstellar extinction law using a least square fit based on $\chi^{2}$ minimisation whose intercept and slope yield the values of $\Delta \mu_{0, i}$ and $\Delta E(B-V)_{i}$, respectively along with their errors (Freedman 1988; Freedman \& Madore 1990; Rich et al. 2014; Scowcroft et al. 2016). Substitutions of these values in equation (3) give the much improved PL relations with reduced dispersions for iteration 2. The solutions for the present sample of FU and FO Cepheids are obtained separately. Once the distances are derived using the PL relations, they are combined together with their corresponding equatorial coordinates $(\alpha, \delta)$ to obtain the Cartesian three dimensional distribution of these Cepheids with respect to the center of the LMC. In order to derive the geometrical and viewing angle parameters, we have selected only those stars which are within $3 \sigma$ limit from the mean of the resulting distance distributions. This ensures that the derived geometrical and viewing angle parameters of the LMC are unaffected by the distance outliers. Out of a total of 3614 stars, 3540 stars have been selected based on the $3 \sigma$ limit which accounts for $98 \%$ of the stars in the sample to find the geometrical and viewing angle parameters of the LMC. Of the remaining 74 stars, 67 lie within $40 \mathrm{kpc}$ and belong to the foreground objects of the LMC. Rest of the 7 stars are the background Cepheids of the LMC located at distances of more than $60 \mathrm{kpc}$. Some of these outliers may be the tracers of lensing populations towards the LMC. 


\section{MULTI-WAVELENGTH PL RELATIONS FOR CLASSICAL CEPHEIDS}

The results of PL relations obtained using the methodology as described in Section 3 are listed in Tables 1 and 2 for FU and FO Cepheids, respectively. Both the iterations, with and without distance/reddening correction are listed. The resulting plots thus obtained from the analysis are shown in Figs. 1 and 2. The observed dispersions of the PL relations as in iteration 1 are due to the four factors: (1) intrinsic variation due to the $\epsilon_{\lambda}$ term in equation (2), (2) distance (3) reddening and (4) photometric errors. In iteration 2 of the fitted PL relations, distance and reddening are corrected and the observed dispersions get smaller after the corrections which account for the intrinsic variation due to the $\epsilon_{\lambda}$ terms as well as photometric errors in the observed magnitudes in the given photometric bands. It should be noted from the plots of the in Figs. 1 and 2 that the dispersions of the observed magnitudes in the left panels are more for the optical and decrease as we go towards longer wavelengths (See third columns of Tables 1 and 2). This is because interstellar extinction is more in the optical and decreases as one goes towards near and mid-infrared bands. However, from the right panels of Figs. 1 and 2, we see the contrast behaviour where the observed magnitudes are corrected for distances and interstellar extinction. The dispersions increase as we go from the optical to the near and mid-infrared in which the observed magnitudes are corrected for distances and interstellar extinction. This is due to the fact that as the wavelength increases, the amplitudes of the Cepheid light curves decrease and hence it becomes increasingly difficult to derive their mean magnitudes with precision.

The PL relations corrected for extinction using the Zaritsky et al. (2004) extinction map obtained for the LMC FU Cepheids derived by Ngeow et al. (2009a) based on the OGLE-III data are consistent with the relations found in the present study. We also find that the respective PL relations obtained by Inno et al. (2016) using the reddening correction determined from the simultaneous multi-wavelength fitting of apparent distance modulus as well as making use of the Haschke et al. (2011) extinction map for the LMC are consistent with the relations derived here within the quoted uncertainties. However, the number of stars varied from 1112 to 1526 while deriving the PL relations in the study of Inno et al. (2016). Unlike the use of different number of FU Cepheids as in the study of PL relations by Inno et al. (2016), we have used the same number of stars (2112) in all the seven photometric bands to derive the PL relations in their respective bands. The dispersions in all the PL relations reduce significantly when corrected for distance and reddening values obtained from the simultaneous solutions of apparent distance moduli calculated in seven photometric bands for individual Cepheids. This reduction in the dispersion for many of the PL relations discussed here is remarkably more than those obtained by Inno et al. (2016). For instance, the reduction in the dispersion of the PL relations for the highly accurate and precise optical OGLE-IV V- and Iband data is more than a factor of two as compared to those obtained by Inno et al. (2016). This fact proves that the reddening values determined in the present study are robust and supports the evidence of a highly accurate reddening map of the LMC. However, because of increasing uncertainties associated with the determinations of the near-infrared mean magnitudes, the dispersions turn out to be of the same order of magnitudes in the present study as those obtained by Inno et al. (2016) in these bands.

One of the important results of the analysis of the present sample of FU Cepheids is the mid-infrared PL relations at [3.6] $\mu \mathrm{m}$ and [4.5] $\mu \mathrm{m}$ bands. The slopes of the two relations obtained in this study $-3.222 \pm 0.007$ and $-3.194 \pm$ 0.006 are comparable to the slopes of $-3.253 \pm 0.010$ and $-3.214 \pm 0.010$ obtained for the two bands by Ngeow et al. (2009a) utilising more than 1600 FU Cepheids based on the IRAC-band data taken from the SAGE Winter'08 Archive. On the other hand, based on the argument that the midinfrared luminosity lies at the Rayleigh-Jeans tail of the blackbody function, the predicted slopes of the PL relations at the mid-infrared will be $\sim-3.23$ (Neilson et al. 2010) which is quite close to the slopes for the two mid-infrared bands used in the present study. The slope of $-3.194 \pm 0.007$ obtained by Inno et al. (2016) with the mean magnitudes determined from the ALLWISE multi-epoch catalog is also in good agreement with the slope $-3.222 \pm 0.007$ for [3.6] $\mu \mathrm{m}$ PL relation obtained from the single-epoch SAGE catalog in the present study.

For the LMC FO Cepheids, it can be seen that the PL relations obtained by Inno et al. (2016) for six multiwavelength photometric bands when corrected for extinction using the reddening values determined from the reddening law fitting method for individual FO Cepheids are drastically different than those obtained using the Haschke et al. (2011) reddening map (For difference between the two PL relations obtained for the same photometric band, see Table 8 of Inno et al. (2016) for FO Cepheids). For example, the PL slopes in the $V$-band when extinction corrections are applied using the reddening values obtained from these two different means are $-3.080 \pm 0.003$ and $-3.299 \pm 0.004$, respectively. These two values are significantly different from each other at more than $6 \sigma$ level. Such huge differences in the PL slopes for other photometric bands are also quite noticeable when extinction corrections were applied using these two different means of reddening estimations in the study of Inno et al. (2016). It is also quite interesting to see that the slope of the PL relation for FO Cepheids in the $V$-band, -3.299 obtained from the extinction correction using the Haschke et al. (2011) reddening map is exactly the same as that obtained Bhardwaj et al. (2016b) which has also been obtained using the same reddening map of Haschke et al. (2011). Nonetheless, this discrepancy raises a serious concern regarding the use of reddening values obtained from various sources while deriving the extinction corrected PL relations for FO Cepheids.

There are two possible reasons why the slopes of the extinction corrected PL relations differ by large values. Firstly, due to the use of different numbers of FO Cepheids by Inno et al. (2016) to derive the PL relations while corrected for extinction using these two sources of reddening estimations. The number of Cepheids used to derive the extinctioncorrected $V$-band PL relation by Inno et al. (2016) using the reddening values obtained from the reddening law fitting method and the Haschke reddening map were 1056 and 795 in number with the dispersions of $\sigma=0.09$ and 0.19 , respectively. Secondly, due to the different levels of uncertain- 
Table 1. Fitted PL relations for the LMC FU Cepheids in the multi-wavelength photometric bands $\{V, I, J, H, K s,[3.6]$, [4.5]\}. Iterations 1 and 2 refer to the PL relations without and with the observed magnitudes corrected for distances and reddenings, respectively.

\begin{tabular}{|c|c|c|c|c|}
\hline Band & $\alpha_{\lambda}$ & $\beta_{\lambda}^{\prime}$ & $\sigma_{\text {fit }}$ & $N$ \\
\hline & \multicolumn{4}{|c|}{ Iteration 1} \\
\hline$V \ldots \ldots \ldots \ldots \ldots$ & $-2.728 \pm 0.095$ & $17.504 \pm 0.062$ & 0.312 & 2112 \\
\hline$I \ldots \ldots \ldots \ldots$ & $-2.954 \pm 0.095$ & $16.866 \pm 0.062$ & 0.218 & 2112 \\
\hline J.............. & $-3.102 \pm 0.095$ & $16.394 \pm 0.062$ & 0.179 & 2112 \\
\hline$H \ldots$ & $-3.203 \pm 0.095$ & $16.113 \pm 0.062$ & 0.175 & 2112 \\
\hline$K s \ldots$ & $-3.226 \pm 0.095$ & $16.052 \pm 0.062$ & 0.178 & 2112 \\
\hline$[3.6] \ldots \ldots \ldots \ldots$ & $-3.224 \pm 0.095$ & $15.929 \pm 0.062$ & 0.201 & 2112 \\
\hline \multirow[t]{2}{*}[4.5]{$\ldots \ldots \ldots \ldots$} & $-3.195 \pm 0.095$ & $15.896 \pm 0.062$ & 0.196 & 2112 \\
\hline & \multicolumn{4}{|c|}{ Iteration 2} \\
\hline$V \ldots$ & $-2.728 \pm 0.003$ & $17.504 \pm 0.002$ & 0.031 & 2112 \\
\hline$I \ldots \ldots \ldots \ldots$ & $-2.954 \pm 0.003$ & $16.865 \pm 0.002$ & 0.035 & 2112 \\
\hline$J \ldots$ & $-3.101 \pm 0.005$ & $16.392 \pm 0.004$ & 0.057 & 2112 \\
\hline$H \ldots$ & $-3.202 \pm 0.006$ & $16.111 \pm 0.004$ & 0.066 & 2112 \\
\hline$K s \ldots \ldots \ldots \ldots$ & $-3.224 \pm 0.008$ & $16.051 \pm 0.005$ & 0.081 & 2112 \\
\hline$[3.6] \ldots \ldots \ldots \ldots$ & $-3.222 \pm 0.007$ & $15.927 \pm 0.004$ & 0.070 & 2112 \\
\hline$[4.5] \ldots$ & $-3.194 \pm 0.006$ & $15.894 \pm 0.004$ & 0.066 & 2112 \\
\hline
\end{tabular}

Table 2. Fitted PL relations for the LMC FO Cepheids in the multi-wavelength photometric bands $\{V, I, J, H, K s,[3.6]$, [4.5]\}. Iterations 1 and 2 refer to the PL relations without and with the observed magnitudes corrected for distances and reddenings, respectively.

\begin{tabular}{|c|c|c|c|c|}
\hline Band & $\alpha_{\lambda}$ & $\beta_{\lambda}^{\prime}$ & $\sigma_{\text {fit }}$ & $N$ \\
\hline & \multicolumn{4}{|c|}{ Iteration 1} \\
\hline$V \ldots \ldots \ldots \ldots \ldots$ & $-3.171 \pm 0.124$ & $17.022 \pm 0.044$ & 0.339 & 1502 \\
\hline$I \ldots \ldots \ldots \ldots$ & $-3.252 \pm 0.124$ & $16.372 \pm 0.044$ & 0.231 & 1502 \\
\hline J............. & $-3.294 \pm 0.124$ & $15.885 \pm 0.044$ & 0.193 & 1502 \\
\hline$H \ldots \ldots \ldots \ldots$ & $-3.342 \pm 0.124$ & $15.604 \pm 0.044$ & 0.187 & 1502 \\
\hline$K s \ldots \ldots \ldots \ldots$ & $-3.284 \pm 0.124$ & $15.520 \pm 0.044$ & 0.219 & 1502 \\
\hline$[3.6] \ldots \ldots \ldots \ldots$ & $-3.295 \pm 0.124$ & $15.416 \pm 0.044$ & 0.196 & 1502 \\
\hline \multirow[t]{2}{*}[4.5]{$\ldots \ldots \ldots \ldots$} & $-3.329 \pm 0.124$ & $15.404 \pm 0.044$ & 0.203 & 1502 \\
\hline & \multicolumn{4}{|c|}{ Iteration 2} \\
\hline$V \ldots \ldots \ldots \ldots \ldots$ & $-3.172 \pm 0.004$ & $17.022 \pm 0.001$ & 0.029 & 1502 \\
\hline$I \ldots \ldots \ldots \ldots \ldots$ & $-3.251 \pm 0.004$ & $16.372 \pm 0.002$ & 0.037 & 1502 \\
\hline$J \ldots$ & $-3.292 \pm 0.010$ & $15.884 \pm 0.004$ & 0.079 & 1502 \\
\hline$H \ldots \ldots \ldots \ldots$ & $-3.339 \pm 0.010$ & $15.603 \pm 0.004$ & 0.084 & 1502 \\
\hline$K s \ldots \ldots \ldots \ldots$ & $-3.280 \pm 0.015$ & $15.519 \pm 0.005$ & 0.120 & 1502 \\
\hline$[3.6] \ldots$ & $-3.292 \pm 0.010$ & $15.415 \pm 0.003$ & 0.077 & 1502 \\
\hline$[4.5]$. & $-3.326 \pm 0.011$ & $15.403 \pm 0.004$ & 0.086 & 1502 \\
\hline
\end{tabular}

ties in the reddening values obtained from these two sources. Comparison with the $V$-band PL slope from Bhardwaj et al. (2016b) rules out the first option, in which the same value as that of Inno et al. (2016) having a dispersion of $\sim 0.18$ was obtained using 1084 number of Cepheids where the reddening correction was done with the help of the Haschke reddening map. Hence we are left only with the second option, i.e., the significant steeper slopes of the PL relations obtained from the Haschke reddening map than those obtained from the reddening law fitting method by Inno et al. (2016) or in the present study are due to large uncertainties in the reddening values of individual stars obtained from the
Haschke reddening map. In fact, for many of the stars we find that the errors in the reddening values obtained from the Haschke et al. (2011) reddening map are of the order of magnitude of the reddening values themselves or sometimes more. For an instance, the average value of the reddening in the Haschke et al. (2011) reddening map is $\sim 0.09$ mag, whereas the average value of the reddening errors is $\sim 0.10 \mathrm{mag}$. Therefore, for the individual Cepheids that have dominating reddening uncertainties, their dereddened magnitudes will have large uncertainties. Nonetheless, we find that the Haschke et al. (2011) reddening map is quite 
consistent with the reddening map obtained in the present study.

It should be noted that reddening causes more dispersions of the PL relations in the optical bands and less to the infrared and mid-infrared bands. From the PL relations obtained for the FO Cepheids as given in Table 2, one can find that the dispersion of the $V$-band PL relation reduces from 0.339 to 0.029 when the extinction corrections are applied using the reddening values obtained from the fitting of the observed apparent distance moduli in seven multiwavelength photometric bands as a function of inverse wavelength in the present study. This demonstrates the fact that accurate values of reddening estimations will cause the dispersions of all the PL relations to reduce significantly while inaccurate values will make a slight reduction in the dispersions and may lead to systematic biases in the PL slopes. The Haschke et al. (2011) reddening map was constructed from the Red Clump stars which are of intermediate-age (ages $\approx 2-9 \mathrm{Gyr}$ ) as compared to the young stellar populations such as classical Cepheids (ages $\approx 10-300 \mathrm{Myr}$ ) used in the present study. Just as it has been seen that applying extinction maps from young OB stars in the LMC to older Red Clump stars in the same regions yields incorrect distances by $0.2 \mathrm{mag}$, it may also be inaccurate to find reddening values on a star-by-star basis for individual Cepheids using reddenings derived from Red Clump stars. Dust affects different stellar populations differently (Zaritsky 1999; Subramaniam 2005). Besides the multi-wavelength photometric data available for Cepheids in more than one band as in the present study is very much suitable to directly determine the reddening values for individual stars rather than relying on various extinction maps. This is particularly useful when the targets are affected due to the high foreground and spatially variable reddening.

\section{REDDENING MAP OF THE LMC}

The values of the true distance modulus $\Delta \mu_{0, i}$ and $\Delta E(B-$ $V)_{i}$ for a representative sample of eight stars in the present analysis is shown in Fig. 3. The mutiband apparent distance moduli for seven photometric bands as a function of inverse wavelength $x=\lambda^{-1}$ are plotted. A reddening law (Cardelli et al. 1989) is then fitted simultaneously to the data which yields the extinction corrected distance modulus $\Delta \mu_{0, i}$ and reddening $\Delta E(B-V)_{i}$ for a particular star. A plot of $\Delta E(B-V)$ vs $\Delta \mu_{0}$ for all Cepheids in the present sample (FU and FO) is shown in Fig. 4. The plot shows that these two values are uncorrelated which reflects an independent and unbiased determination of these two quantities using the methodology as described in Section 3. Fig. 5 shows the reddening values as a function of the period which addresses the fact that there is no systematic effect on the derived reddening values using the PL relations. The values of distance modulus $\Delta \mu_{i}$ and reddening $\Delta E(B-V)_{i}$ derived from equation (4) are offsets from the mean distance modulus and reddening of the LMC. In the present case, these values are arbitrary and cannot be determined based on the data alone. Since the purpose of the present paper is not to calibrate the mean distance and reddening of the LMC, but to determine its geometrical and structural parameters, using the mean values of these parameters does not have any impact on the results (Nikolaev et al. 2004). Following Nikolaev et al. (2004), we assume $\overline{E(B-V)})_{\text {LMC }}=0.14 \pm 0.02 \mathrm{mag}$ and the mean value of the $\mathrm{LMC}$ distance modulus is taken as $\bar{\mu}_{\mathrm{LMC}}=$ $18.493 \pm 0.002$ (stat.) \pm 0.047 (sys.) mag (Pietrzyński et al. 2013). Therefore, the absolute values of reddening and distance modulus of the present sample of classical Cepheids are obtained from $E(B-V)_{i}=\Delta E(B-V)_{i}+0.14$ mag, $\mu_{0, i}=\Delta \mu_{0, i}+18.493 \mathrm{mag}$. The use of the value of $\overline{E(B-V)}_{\mathrm{LMC}}=0.14 \pm 0.02 \mathrm{mag}$ makes most of the stars in our sample to have $E(B-V)_{i}>0$. For 25 stars, we get $E(B-V)<0$ which constitute less than $1 \%$ of the present sample of classical Cepheids.

Once the absolute reddening values for all the stars are obtained, we proceed towards constructing the reddening map of the LMC. Using the information of $(\alpha, \delta)$ for all the Cepheids as provided in the Inno et al. (2016) catalog, we first bin the observed area of the LMC on a $(10 \times 10)$ coordinate grid. Depending on the $(\alpha, \delta)$ values, each grid will contain a finite number of stars with their absolute reddening values. Reddening distribution of the present sample of LMC classical Cepheids is shown in Fig. 6. The weighted average values of reddening of all the stars falling in a bin is taken to be the value of reddening corresponding to that bin with standard deviation as its statistical uncertainty. From the reddening map, we can see that the reddening values in the region between $\alpha=83^{\circ}-88^{\circ}, \delta=-71^{\circ}$ to $-68^{\circ}$ have the highest values with the most prominent one roughly located at $\alpha \sim 85^{\circ}$ and $\delta=-69^{\circ}$. This region was also identified as having the highest reddening in the study of Nikolaev et al. (2004) as well as Inno et al. (2016) and is associated with the 30 Doradus region (Tarantula Nebula), the most active star forming brightest HII region in the Local group located in the bar of the LMC (Tatton et al. 2013). The 30 Doradus region is located at the center of the compact massive cluster RMC 136a (Evans et al. 2011; Tatton et al. 2013) and contains the highest concentration of young clusters (Glatt et al. 2010). Another region located in the vicinity of $\alpha=74^{\circ}$ and $\delta=-69^{\circ}$ has higher values of reddening. We identify this region to be associated with the LMC HI supergiant shells SGS 12 (LMC3) which hosts young star clusters (Glatt et al. 2010). For better visualisation of the reddening structure, the density maps of reddening for $\mathrm{FO}$, $\mathrm{FU}$ and combined $(\mathrm{FO}+\mathrm{FU})$ Cepheids are shown in Fig. 7. The maps are produced using the IDL routine filter_image by applying $(3 \times 3)$ median and then $(3 \times 3)$ moving average, both applied to all the pixels. The bins with number of stars $\geq 3$ are used in order to reduce the noise in the reddening density map. The reddening map for the LMC obtained by Nikolaev et al. (2004) and Inno et al. (2016) using the classical Cepheids are in good agreement with the map found in the present study, although the number of Cepheids as well as the number of wavelength bands were less in earlier studies. The map is also consistent with the Haschke et al. (2011) reddening map obtained using the Red Clump stars.

We have also quantified how the reddening and distance values change if there were no mid-infrared photometry. The mean and standard deviation of the difference in the two values of $\Delta E(B-V)$ obtained with and without the midinfrared photometry are 0.00 and 0.03 mags, respectively, whereas the corresponding values for distance are $0.01 \mathrm{kpc}$ and $1.68 \mathrm{kpc}$, respectively. Therefore, the individual reddening and distance values can differ by $0.03 \mathrm{mag}$ and $1.68 \mathrm{kpc}$, 

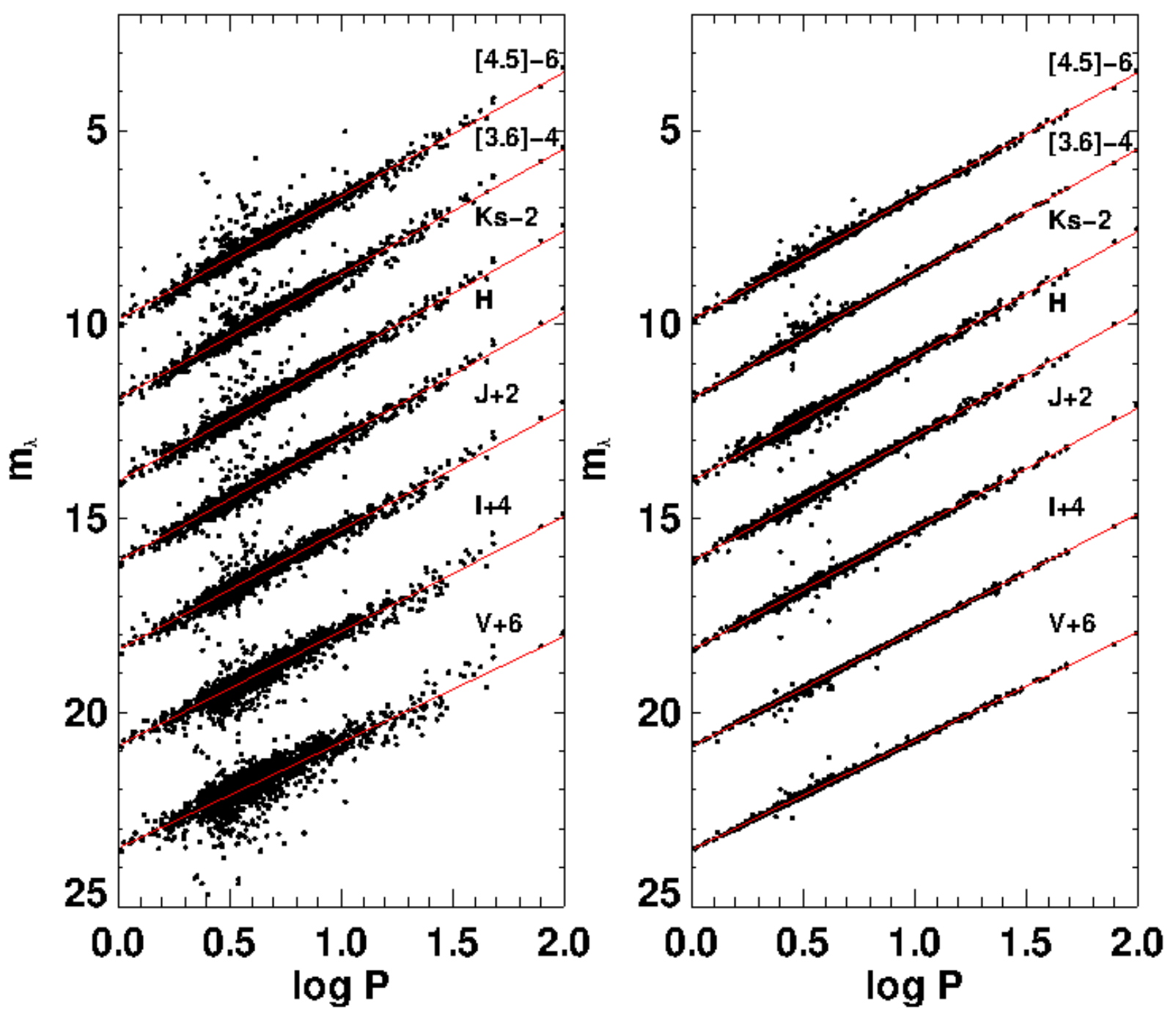

Figure 1. Leavitt law for FU Cephieds. The left panel shows the fitted PL relations to the observed magnitudes obtained using iteration 1. The right panel shows fits to the observed magnitudes obtained in iteration 2 corrected for distance and reddening found from iteration 1.

respectively at the $1 \sigma$ uncertainty level. However, the means of these values in both the two cases are almost identical.

\section{THREE DIMENSIONAL STRUCTURE OF THE LMC}

A common objective in studying the three dimensional structure of a galaxy is to find its angular orientation and the axes ratios in three different directions. The orientation of the galaxy is measured by means of two angles, the inclination angle $(i)$, and the position angle of the line of nodes $\left(\theta_{l o n}\right)$. The angle $\theta_{l o n}$ is defined as the angle between the intersection of the plane of the distribution of the stars in the galaxy and the sky plane defined in Cartesian coordinates. Position angle in astronomy is measured from north $\left(0^{\circ}\right)$ towards east $90^{\circ}$. The inclination angle $(i)$ is defined as the angle how the galaxy plane $\left(x^{\prime}, y^{\prime}\right)$ is inclined with respect to the sky plane $(x, y)$ (van der Marel \& Cioni 2001). The combination of these two angles gives a full description of the orientation of a galaxy, and combined with its $(\alpha, \delta)$ values and distance $(D)$, a full description of its position in three dimensional space can be obtained. The orientation of a galaxy is an important parameter in galaxy interaction simulations. The most commonly used method to obtain the angles $i$ and $\theta_{l o n}$ is by fitting a plane of the form $z=f(x, y)$ to the distribution of the stars in Cartesian coordinates $(x, y, z)$, and finding these angles between that plane and the sky plane. To a reasonable limit, the geometry of the LMC can be considered to be planar (van der Marel \& Cioni 2001). Another method, which has recently come into use, is to fit a triaxial ellipsoid to the Cartesian distribution 

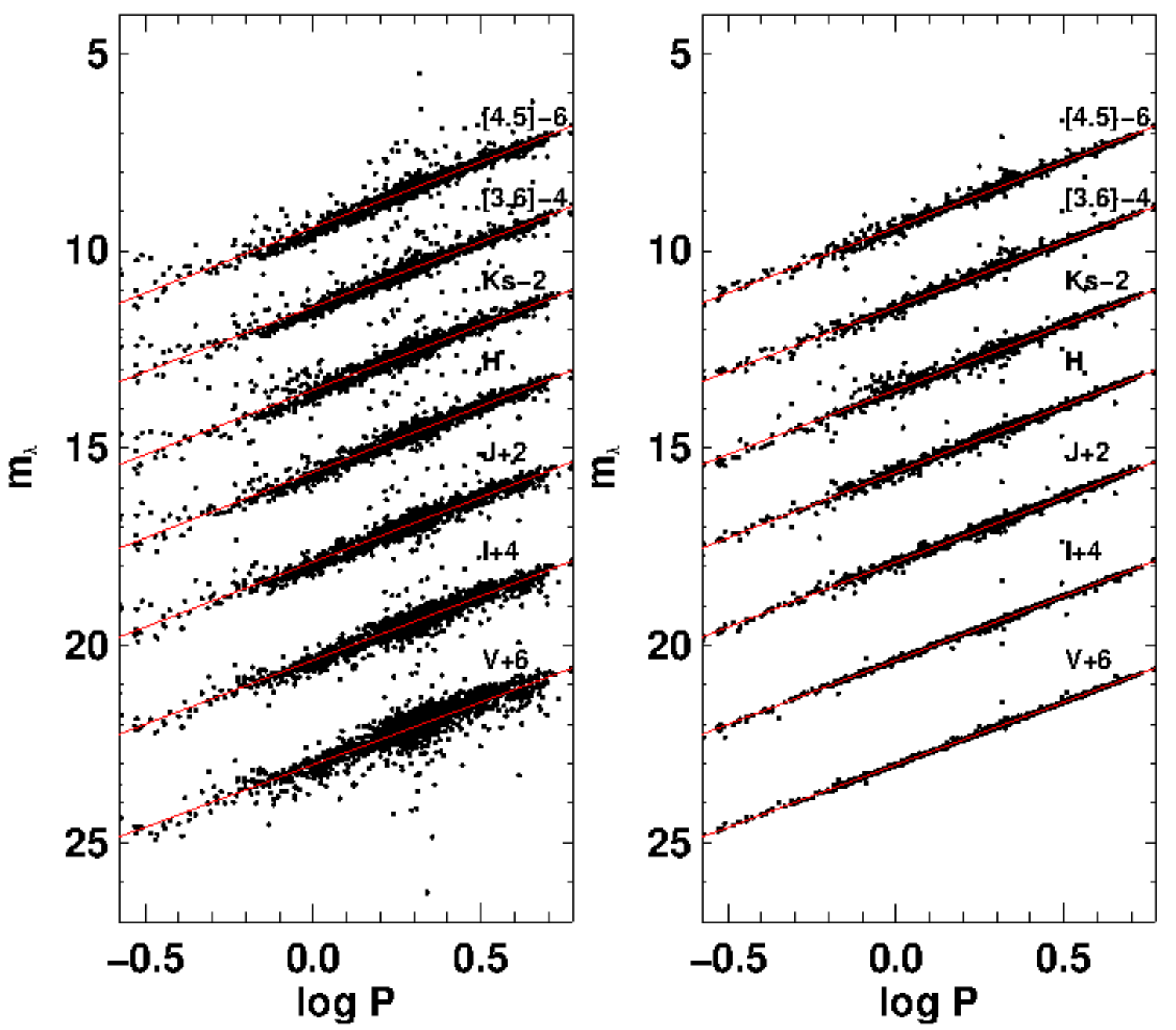

Figure 2. Leavitt law for FO Cephieds. The left panel shows the fitted PL relations to the observed magnitudes obtained using iteration 1. The right panel shows fits to the observed magnitudes obtained in iteration 2 corrected for distance and reddening found from iteration 1.

of the stars, by means of a principal axis transformation. Apart from finding these two angles, this method also yields the values of the axes ratios of the galaxy in three perpendicular directions (Paz et al. 2006; Pejcha \& Stanek 2009; Subramanian \& Subramaniam 2012; Deb \& Singh 2014).

The Inno et al. (2016) catalog gives the $(\alpha, \delta)$ values for individual stars, and the distances $(D)$ are obtained through the period-luminosity relations, as described in Section 4. The values of these quantities $(\alpha, \delta, D)$ are transformed into the Cartesian coordinate system $(x, y, z)$, where the $z$-axis is pointed towards the observer, the $x$-axis is antiparallel to the $\alpha$-axis, and the $y$-axis is parallel to the $\delta$-axis. $D_{0}$ is the distance between the center of the target galaxy and the observer, while $D$ is the distance to a particular star, and $\left(\alpha_{0}, \delta_{0}\right)$ is the center of the target galaxy in equatorial coordinates. The center of the LMC in the present study is taken as $\left(\alpha_{0}, \delta_{0}\right)=\left(80^{\circ} .78,-69^{\circ} .03\right)$ (Nikolaev et al. 2004) and the distance to the center is taken as $D_{0}=49.973 \mathrm{kpc}$ (Pietrzyński et al. 2013). The transformation from $(\alpha, \delta, D)$ to $(x, y, z)$ can be performed by the transformation equations

$x=-D \sin \left(\alpha-\alpha_{0}\right) \cos \delta$,

$y=D \sin \delta \cos \delta_{0}-D \sin \delta_{0} \cos \left(\alpha-\alpha_{0}\right) \cos \delta$,

$z=D_{0}-D \sin \delta \sin \delta_{0}-D \cos \delta_{0} \cos \alpha-\alpha_{0} \cos \delta$.

The coordinate system of the LMC $\left(x^{\prime}, y^{\prime}, z^{\prime}\right)$ is the same as the coordinate system $(x, y, z)$, except that it has been rotated counterclockwise by an angle $\theta$ about the $z$-axis, and then clockwise by an angle $i$ about the new $x$-axis. These 

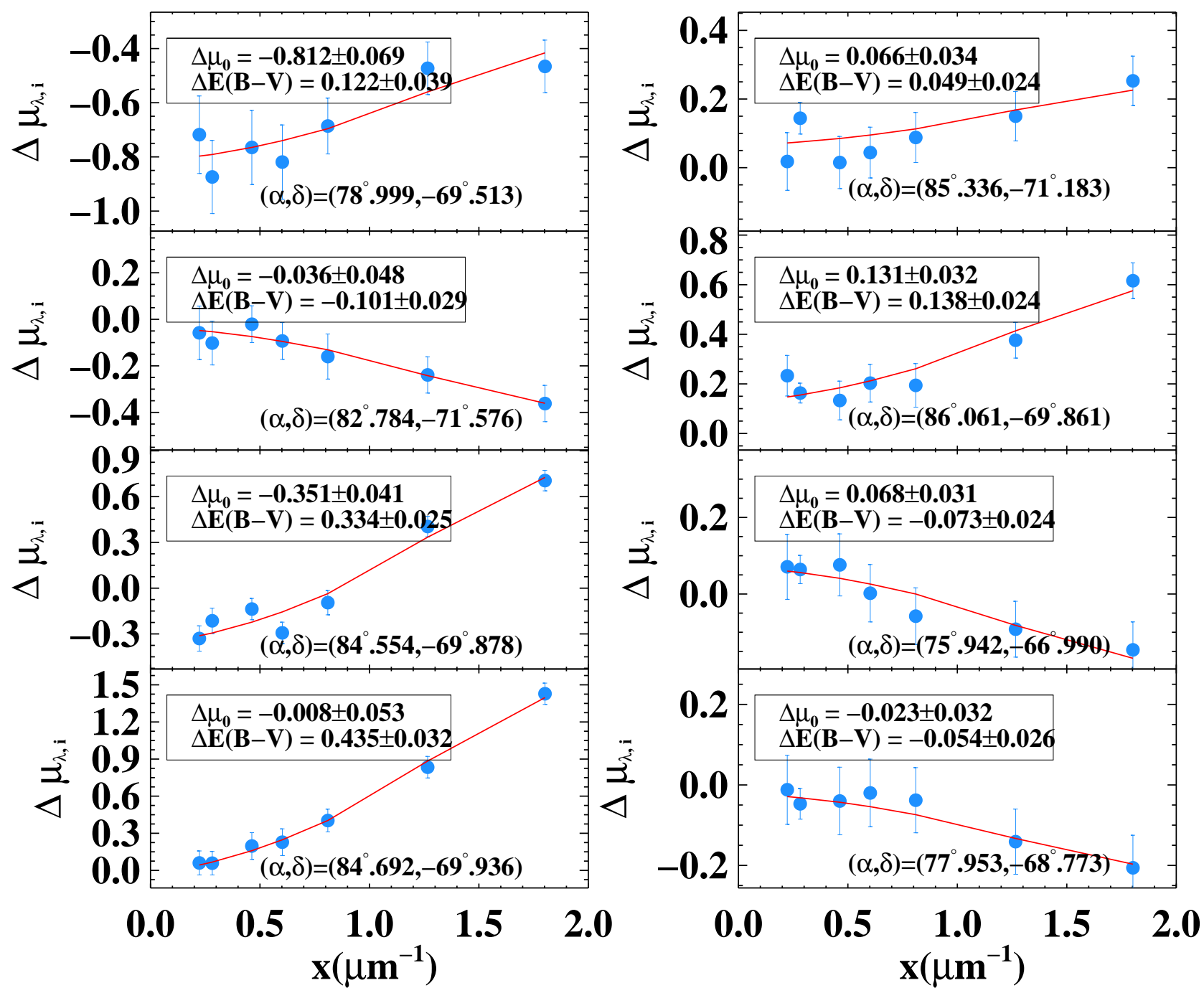

Figure 3. The method of calculation of true distance modulus $\Delta \mu_{0, i}$ and reddening $\Delta E(B-V)_{i}$. Each of the data points corresponds to the distance modulus $\Delta \mu_{\lambda, i}$ determined for the seven photometric bands plotted as a function of inverse of their respective wavelengths $\left(\lambda^{-1}\right)$. A reddening law (Cardelli et al. 1989) is fitted simultaneously to the seven distance moduli using the PL relations obtained from iteration 1 as listed in Tables 1 anf 2 based on the $\chi^{2}$ minimisation. The solid line shows the best fit reddening law which yields the extinction corrected distance modulus $\Delta \mu_{0, i}$ and reddening $\Delta E(B-V)_{i}$.

coordinate transformations can be written as

$$
\left[\begin{array}{l}
x^{\prime} \\
y^{\prime} \\
z^{\prime}
\end{array}\right]=\left[\begin{array}{ccc}
\cos \theta & \sin \theta & 0 \\
-\sin \theta \cos i & \cos \theta \cos i & -\sin i \\
-\sin \theta \sin i & \cos \theta \sin i & \cos i
\end{array}\right]\left[\begin{array}{l}
x \\
y \\
z
\end{array}\right]
$$

The Cartesian projected distributions of the LMC Cepheids are shown in Fig. 8. Let us denote the transformation matrix as

$T=\left[\begin{array}{ccc}\cos \theta & \sin \theta & 0 \\ -\sin \theta \cos i & \cos \theta \cos i & -\sin i \\ -\sin \theta \sin i & \cos \theta \sin i & \cos i\end{array}\right]$.

\subsection{Moment of Inertia Tensor Analysis}

Distribution of Cepheids in the LMC can be modeled as a rigid body whose inertial properties can be completely described by the inertia ellipsoid obtained analogously from the moment of tensor of rigid bodies. Let the reference coordinates of the sky plane be defined by Cartesian coordinates $(x, y, z)$. The orientation of the galaxy with respect to the the plane of the sky can be described by an ellipsoid given by the rotated or transformed Cartesian coordinates $\left(x^{\prime}, y^{\prime}, z^{\prime}\right)$ whose centroid coincides with the origin of the coordinate system $(x, y, z)$. The transformed or rotated coordinate system $\left(x^{\prime}, y^{\prime}, z^{\prime}\right)$ is obtained from the diagonalisation of the inertia tensor $\mathbf{I}$. The inertia tensor, $\mathbf{I}$, can be constructed as 


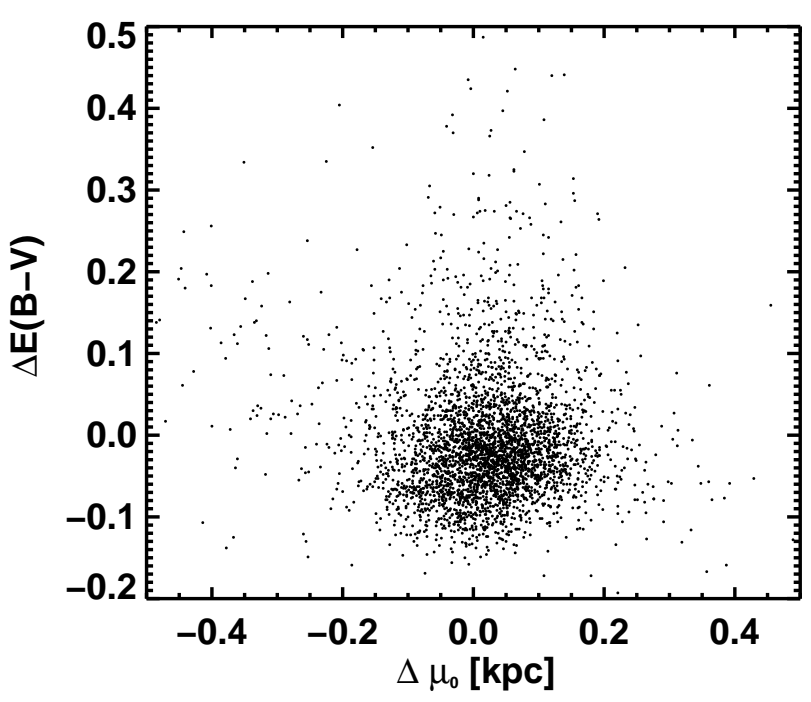

Figure 4. $\Delta E(B-V)$ vs $\Delta \mu_{0}$ for all Cepheids in the present sample (FU and FO). These values are found to uncorrelated which reflects the independent and unbiased determination of these two quantities using the methodology as described in Section 3.

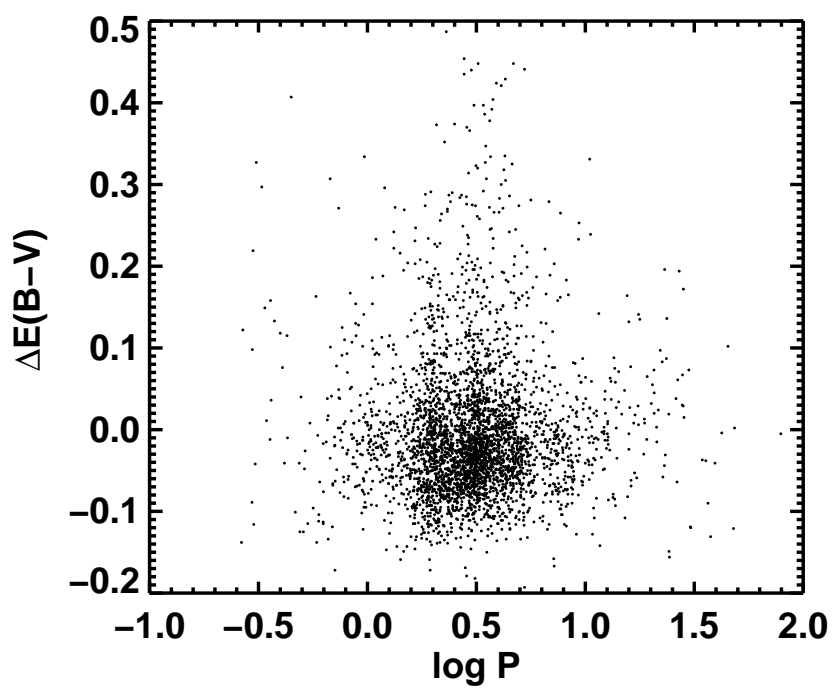

Figure 5. Reddening values as a function of the logarithm of period. The plot suggests that there is no systematic error due to the given PL relations.

follows (Deb \& Singh 2014):

$\mathbf{I}=\left[\begin{array}{ccc}I_{x x} & -I_{x y} & -I_{x z} \\ -I_{y x} & I_{y y} & -I_{y z} \\ -I_{z x} & -I_{z y} & I_{z z}\end{array}\right]$

where the components are defined by

$I_{\alpha \beta}= \begin{cases}\sum_{\gamma \neq \alpha} \operatorname{Cov}(\gamma, \gamma), & \text { if } \alpha=\beta \\ \operatorname{Cov}(\alpha, \beta), & \text { if } \alpha \neq \beta\end{cases}$ where covariance is defined by

$\operatorname{Cov}(\alpha, \beta)=\frac{1}{N} \sum_{i=1}^{N}(\alpha(i)-\bar{\alpha})(\beta(i)-\bar{\beta})$,

and the centroid is defined by

$\bar{\alpha}=\sum_{i=1}^{N} \alpha(i) / N$.

The moment of inertia tensor $\mathbf{I}$ by construction itself is a symmetric matrix and hence can be easily diagonalised. The axes of the coordinate system $(x, y, z)$ are selected in such a way that $I_{x y}=I_{x z}=I_{y z}=0$ giving rise to the principal moments of inertia of the system given by $I_{x x}, I_{y y}$ and $I_{z z}$, respectively. This can be obtained from the diagonalisation of the symmetric covariance matrix $\mathbf{I}$ which is achieved through a rotation or transformation matrix $\mathbf{T}$. The matrix $\mathbf{T}$ is formed with the eigenvectors of $\mathbf{I}$ as its column vectors. The matrix $\mathbf{T}$ therefore serves as the rotation or transformation matrix to the new coordinate system, $T: \mathbb{R}^{3} \rightarrow \mathbb{R}^{\prime 3}$. It can be seen that the matrix $\mathbf{T}^{-1} \mathbf{I T}$ is a diagonal matrix whose diagonal elements are the eigenvalues of the covariance matrix I. The individual eigenvectors represent the three orthogonal axes in the new coordinate system $\left(x^{\prime}, y^{\prime}, z^{\prime}\right)$, and are called the principal axes. Three eigenvalues $\left(\lambda_{1}>\lambda_{2}>\lambda_{3}\right)$ correspond to these three eigenvectors, and are called the principal moments of inertia of the system. The principal axes with the smallest, intermediate and largest eigenvalues are often called the minor, intermediate and major axes, respectively of the inertia ellipsoid. The lengths of the semi-axes of the ellipsoid are given by (Deb \& Singh 2014)

$S_{i}=\sqrt{\frac{5}{2}\left(\lambda_{j}+\lambda_{k}-\lambda_{i}\right)}$, for $i \neq j \neq k$.

The transformation matrix or the rotation matrix $(\mathbf{T})$ of the transformation $T: R^{3} \rightarrow R^{\prime 3}$ obtained from the eigenvectors of the matrix $\mathbf{I}$ as column vectors is given by

$\left(\begin{array}{l}e_{1}^{\prime} \\ e_{2}^{\prime} \\ e_{3}^{\prime}\end{array}\right)=\left(\begin{array}{lll}T_{11} & T_{21} & T_{31} \\ T_{12} & T_{22} & T_{32} \\ T_{13} & T_{23} & T_{33}\end{array}\right)\left(\begin{array}{l}e_{1} \\ e_{2} \\ e_{3}\end{array}\right)$,

where $T_{i j}=\cos \left(e_{i} . e_{j}^{\prime}\right)$ are the direction cosines. $\vec{e}=$ $\left(e_{1}, e_{2}, e_{3}\right)^{T}$ and $\overrightarrow{e^{\prime}}=\left(e_{1}^{\prime}, e_{2}^{\prime}, e_{3}^{\prime}\right)^{T}$ denote the basis vectors in the Cartesian coordinate system $(x, y, z)$ and the new rotated coordinate system $\left(x^{\prime}, y^{\prime}, z^{\prime}\right)$, respectively. The transformation matrix $\mathbf{T}$ contains the information about the orientation of the ellipsoid with respect to the unrotated coordinate system $(x, y, z)$ which can be used to find its position angle $(\theta)$ and inclination angle $(i)$ (Subramanian \& Subramaniam 2012; Deb \& Singh 2014). It should be noted that the position angle $\theta$ as defined in equation (6) is measured from the positive $\mathrm{x}$-axis, i.e., from the west direction. However, in astronomical convention, position angles are always measured from the north $\left(0^{\circ}\right)$ towards east $\left(90^{\circ}\right)$ (van der Marel \& Cioni 2001). Therefore, the value of $\theta$ as measured with the usual astronomical convention will be given by $\theta_{\text {lon }}=\theta+90^{\circ}$. Also, since the line of nodes is a line its value can be described by two different position angles that differ by $180^{\circ}$ (van der Marel \& Cioni 2001).

Application of the moment of inertia tensor analysis 

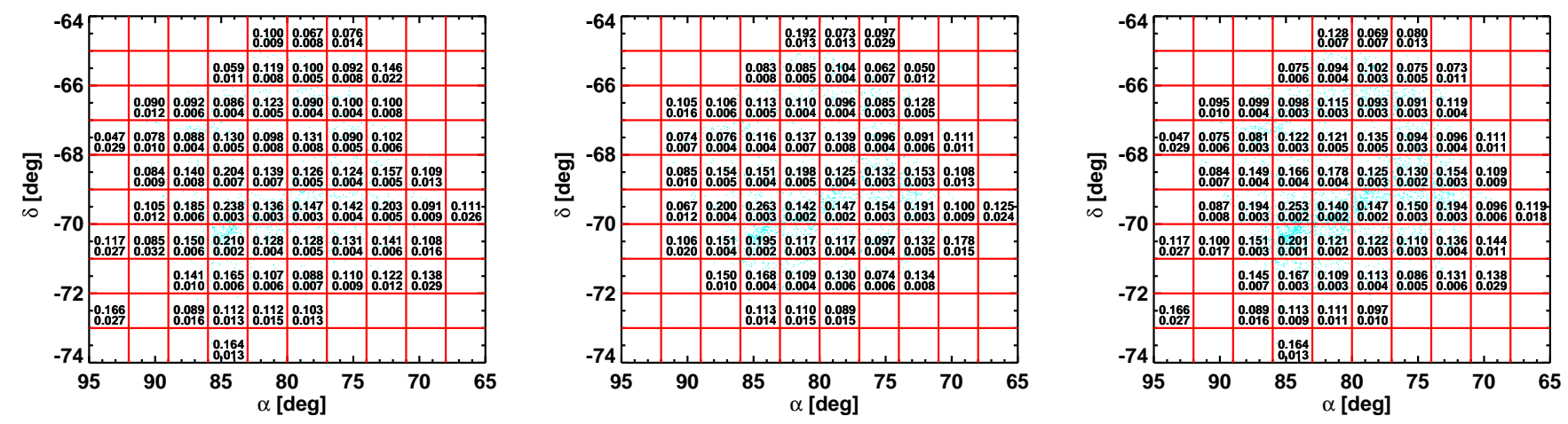

Figure 6. Reddening distribution $E(B-V)$ of the selected sample of FO, FU and (FO+FU) Cepheids in the LMC. $E(B-V)$ values are binned on a $10 \times 10$ coordinate grid. Weighted average reddening values and their associated errors calculated are shown in each grid box.
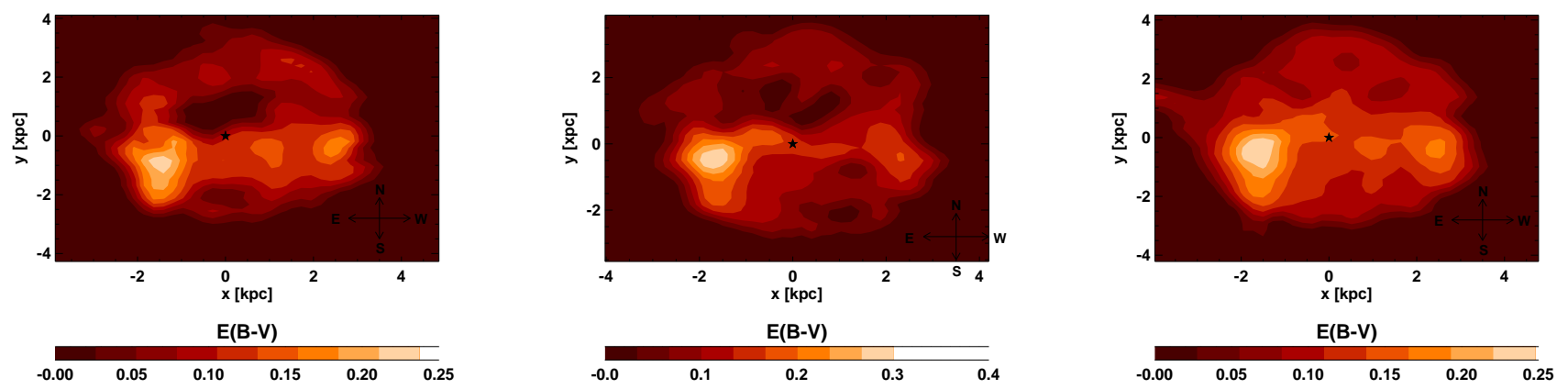

Figure 7. Density maps of reddening using FO, FU and combined (FO+FU) Cepheids are shown from left to right. The map is produced computing the average reddening on a $40 \times 40$ grid in $(x, y)$ coordinates and smoothing the resulting distribution using the IDL routine filter_image. In all the panels a high reddening zone near 30 dor $(x \sim-1.8, y \sim-1)$ is quite easily discernible.
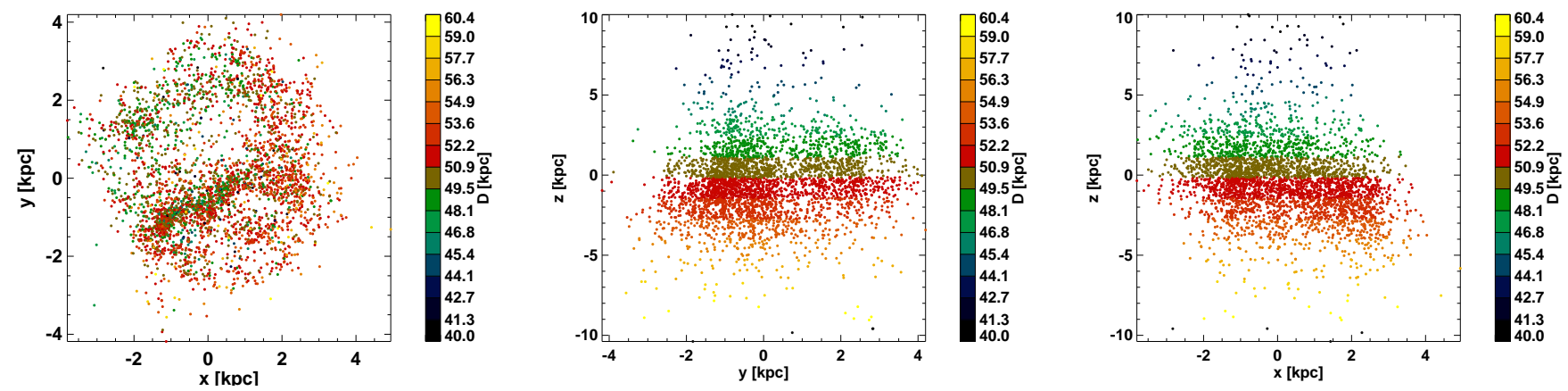

Figure 8. The projected distribution of LMC Cepheids on the $X Y, Y Z$ and $X Z$ planes with the colour bar plots of their distances.

to the $(x, y, z)$ distribution of the classical Cepheids without considering the errors $\left(\sigma_{x}, \sigma_{y}, \sigma_{z}\right)$ yields the eigenvalues $\lambda_{1}=7.874, \lambda_{2}=7.224$ and $\lambda_{3}=4.182$. Using the transformation matrix constructed from the eigenvectors corresponding to these eigenvalues, the values of $\theta$ and $i$ are $15^{\circ} .858$ and $38^{\circ} .953$, respectively. However, the value of $\theta$ obtained here is measured with respect to the $x$-axis (west). If measured according to the astronomical convention from the positive $y$-direction (north), it comes out to be $\theta_{\text {lon }}=128^{\circ} .953$. The final values of these parameters along with their errors are obtained using Monte Carlo simulations as described in Deb et al. (2015). In order to carry out the Monte Carlo simulations, we have randomly generated Cartesian distributions $(x, y, z)$ using the errors 
$\left(\sigma_{x}, \sigma_{y}, \sigma_{z}\right)$ from the observed distributions. The procedure of modeling the randomly generated distribution of stars is done with the help of the moment of tensor analysis for $10^{5}$ iterations. In each iteration of the Monte carlo simulations, the values of the parameters of the ellipsoid such as $\left\{i, \theta_{\text {lon }}, S_{0}, S_{1}, S_{2}\right\}$ are noted down. Monte Carlo simulations for $10^{5}$ iterations helps to build up the distribution of these five parameters. The histograms obtained from the individual distributions of each of the five parameters are fitted with a three-parameter Gaussian distribution which yield the respective values $\mu, \sigma$ and the peak of the histogram. From the Monte Carlo simulations, the following values of the parameters are obtained for the LMC with axes ratios $1.000 \pm 0.003: 1.151 \pm 0.003: 1.890 \pm 0.014$ and the viewing angle parameters: inclination angle of $i=11^{\circ} .920 \pm 0^{\circ} .315$ with respect to the longest axis from the line of sight and position angle of line of nodes $\theta_{\text {lon }}=128^{\circ} .871 \pm 0^{\circ} .569$ as measured eastwards from north following astronomical convention. The lengths of the semi-major, intermediate and semi-minor axes for the galaxy are $S_{0}=5.712 \pm 0.038 \mathrm{kpc}$, $S_{1}=3.480 \pm 0.003 \mathrm{kpc}, S_{2}=3.021 \pm 0.008 \mathrm{kpc}$, respectively, where $S_{0}>S_{1}>S_{2}$.

\subsection{Plane fit solution to the $z$ distribution}

Since we have the $(x, y, z)$ distribution for each star in the Cartesian coordinate system, we now derive the inclination $(i)$ and position angle $\left(\theta_{\text {lon }}\right)$ by fitting a plane solution of the following form (Nikolaev et al. 2004):

$z=A x+B y+C$.

The values of $A$ and $B$ obtained from the plane fit solution given by equation (13) are then used to compute the position angle $\left(\theta_{\text {lon }}\right)$ and inclination $(i)$ of the LMC disk:

$$
\begin{gathered}
\theta_{\text {lon }}=\arctan \left(-\frac{A}{B}\right)+\operatorname{sign}(B) \frac{\pi}{2}, \\
i=\arccos \left(\frac{1}{\sqrt{1+A^{2}+B^{2}}}\right) .
\end{gathered}
$$

The standard errors in $\theta$ and $i$ are obtained from the propagation of error formula as follows:

$\sigma_{\theta}=\frac{1}{\sqrt{A^{2}+B^{2}}} \sqrt{A^{2} \sigma_{B}^{2}+B^{2} \sigma_{A}^{2}}$,

$\sigma_{i}=\frac{1}{A^{2}+B^{2}+1} \frac{1}{\sqrt{A^{2}+B^{2}}} \sqrt{A^{2} \sigma_{A}^{2}+B^{2} \sigma_{B}^{2}}$.

The results obtained from the plane fit solution are listed in Table 3 using FO, FU and (FO+FU) Cepheids, respectively. The fitted plane using the parameters obtained from the plane fit using $(\mathrm{FO}+\mathrm{FU})$ Cepheids is shown in Fig. 9. We find that the values of the viewing angle parameters obtained by Inno et al. (2016) are in good agreement with those obtained in the present study although the methodology of obtaining their values in these two studies are completely different. However, we note that the statistical errors of the derived viewing angle parameters as given in Inno et al. (2016) are unrealistically small and seem to be wrongly quoted. The errors quoted should actually be in radians if they are correctly calculated in Inno et al. (2016) and need to be multiplied by $\frac{180}{\pi}$ to convert them into degrees while writing down the results of the measured values. Making this correction, we write down the values obtained by Inno et al.

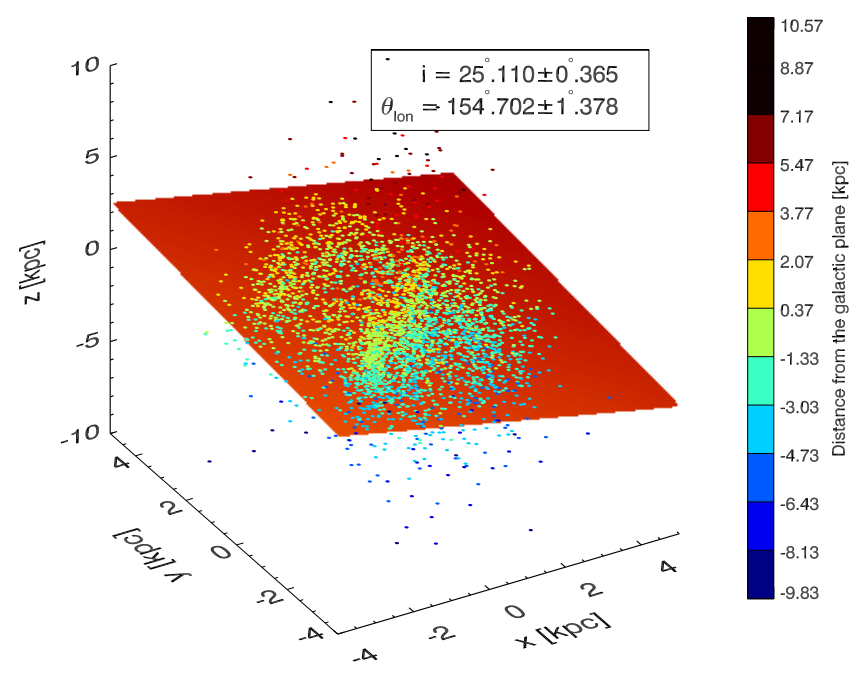

Figure 9. Three dimensional $(x, y, z)$ distribution of the combined $(\mathrm{FO}+\mathrm{FU})$ sample of LMC classical Cepheids. The fitted plane with the parameters obtained from the plane fit is also overplotted.

(2016) as $i=25^{\circ} .05 \pm 1^{\circ} .146, \theta_{\text {lon }}=150^{\circ} .76 \pm 1^{\circ} .146$. Comparing these values with those obtained in the present study (third row of Table 3 ) show that the $\theta_{\text {lon }}$ value differs at the $2.2 \sigma$ level and the $i$ values are consistent within $0.2 \sigma$. On the other hand, the values of $i=26^{\circ} .2 \pm 5^{\circ} .9$ and $\theta_{\text {lon }}=154^{\circ} .5 \pm 2^{\circ} .1$ found by van der Marel \& Kallivayalil (2014) combining the proper motion measurements and lineof-sight velocities for young stars in the LMC are consistent with their values obtained in the present study within $0.2 \sigma$ and $0.1 \sigma$ levels, respectively. The viewing angle parameters obtained in the present study are also comparable to the values of $i=23^{\circ} .5 \pm 0^{\circ} .4$ and $\theta_{\mathrm{lon}}=154^{\circ} .6 \pm 1^{\circ} .2$ obtained by Koerwer (2009) using the Red Clump stars. A comprehensive list of the viewing angle parameters for the LMC available in the literature obtained using various tracers can be found in Inno et al. (2016).

One can easily see that the values of $\left(i, \theta_{\text {lon }}\right)$ obtained from the moment of tensor analysis and the plane fitting procedure differ significantly because these two methods are based on completely different principles. Most of the values of the viewing angle parameters of the LMC as quoted in the literature are based on the plane fitting procedure and hence the comparisons of these values have been done obtained using this method in the present study. However the moment of tensor analysis yields the values of the axes ratios of the LMC apart from finding the viewing angle parameters. This is particularly useful to know the extent of the LMC in three perpendicular directions. 
Table 3. Plane fit solutions to the $z$-distribution of the LMC Cepheids as a function of $(x, y)$ coordinates using FO, FU and (FO+FU) Cepheids. The resulting viewing angle parameters, $i$ and $\theta$ calculated from solutions of the plane fit.

\begin{tabular}{|c|c|c|c|c|}
\hline Solution & $i$ & $\theta_{\text {lon }}$ & $\chi^{2}$ & Remarks \\
\hline$z_{\mathrm{fit}}=(-0.432 \pm 0.016) x+(0.233 \pm 0.015) y+(-0.193 \pm 0.023)$ & $26^{\circ} .151 \pm 0^{\circ} .499$ & $151^{\circ} .614 \pm 1.764$ & 3.884 & FO only \\
\hline$z_{\mathrm{fit}}=(-0.410 \pm 0.017) x+(0.156 \pm 0.017) y+(-0.205 \pm 0.025)$ & $23^{\circ} .691 \pm 0^{\circ} .534$ & $159^{\circ} .206 \pm 2.229$ & 1.662 & FU only \\
\hline$z_{\mathrm{fit}}=(-0.424 \pm 0.011) x+(0.200 \pm 0.011) y+(-0.195 \pm 0.017)$ & $25^{\circ} .110 \pm 0^{\circ} .365$ & $154^{\circ} .702 \pm 1.378$ & 2.586 & $(\mathrm{FO}+\mathrm{FU})$ \\
\hline
\end{tabular}

\section{SEPARATION OF BAR AND DISK OF THE LMC}

The LMC has a prominent luminous central optical bar which is slightly off-centered and misaligned with the disk (Zhao \& Evans 2000). The bar is morphologically peculiar in the sense that the eastern tip of the bar shows an overdensity and is dominated by the patchy distribution of young stars and higher values of dust extinction as seen in optical images (van der Marel \& Kallivayalil 2014). The bar of the LMC has been observed in the distributions of young, intermediate-age and and old stellar populations. The bar is roughly of the $\left(3^{\circ} \times 1^{\circ}\right)$ angular size (Westerlund 1997; Mancini et al. 2004). A number of numerical simulations have been carried out in the recent past to explain the formation of the off-center bar of the LMC. It was suggested by Zhao \& Evans (2000) that the off-center LMC bar is the result of a recent tidal interaction of the LMC with the SMC and the Milky Way. In one of the studies, the offcenter bar of the LMC is shown to be the result of collision of the LMC with a low-mass sub-halo (dark satellites) of mass $10^{8}-10^{9} M_{\odot}$ belonging to the Galaxy from numerical simulations (Bekki 2009). The most recent studies attempt to explain the formation of the bar due to the close encounter of the LMC and SMC (dwarf-dwarf galaxy interaction) a few Myr ago (Besla et al. 2012; Yozin \& Bekki 2014; Pardy et al. 2016; Kruk et al. 2017). Using a sample of 270 late type galaxies from the multi-wavelength Sloan Digital Sky Survey (SDSS) images and Galaxy Zoo morphologies, Kruk et al. (2017) measured offsets in the range $0.2-2.5 \mathrm{kpc}$ between the photometric centres of the stellar disk and stellar bar. Majority of the galaxies with off-centre bars in that study are of Magellanic type and this provides evidences in the support of predictions from the simulations of dwarf-dwarf galaxy interactions. Reconstructing the observed structures of a galaxy from the simulations of galaxy interactions is often quite challenging and provides an insight into the understanding of the evolution of the Universe.

From the study of three dimensional structure of the LMC using different tracers, a number of studies were devoted to finding out whether the bar of the LMC is off-centred and misaligned with respect to its disk (Nikolaev et al. 2004; Koerwer 2009; Subramanian \& Subramaniam 2013; van der Marel \& Kallivayalil 2014; Jacyszyn-Dobrzeniecka et al. 2016). All these studies except the one by Jacyszyn-Dobrzeniecka et al. (2016) found the bar of the LMC to be offset from its disk with the former being closer to the observer than the latter. In the the study of three dimensional structure of the LMC using OGLE classical Cepheids, Jacyszyn-Dobrzeniecka et al.
(2016) redefined the definition of the bar stating that the entire bar should consist of the eastern part as well as extend towards the western end of the LMC contrary to the existing conventional definition of the bar in the literature which is believed to be consisting of only the eastern part of the LMC. Nonetheless, we select the bar region following its conventional definition as in the literature. In order to get an idea about the LMC bar and disk from the present analysis carried out in seven bands using a statistically larger sample of classical Cephieds, we make a slight modification of the definition of the bar following Nikolaev et al. (2004). This is done just to ensure that the dimension of the bar is roughly of the order of $\approx(3 \mathrm{kpc} \times 1 \mathrm{kpc})$ in the $X Y$-plane as viewed along the line of nodes noting that $1^{\circ} \sim 1 \mathrm{kpc}$ at the distance of the LMC. The bar is defined as the rectangular region in the $X Y$-plane using the inequalities

$$
\begin{aligned}
& y>0.5 x-1.0 \\
& y<0.5 x \\
& y>-2 x-5.0 \\
& y<-2 x+2.5
\end{aligned}
$$

Stars satisfying the above inequalities are designated as "bar" Cepheids else as "disk" Cepheids. The location of the bar is overplotted on the two dimensional density contour map of the LMC in Fig. (10). Once the separation between the disk and bar Cepheids is made, we shift the bar and the disk towards the observer along the line of nodes through a clockwise rotation about the $z$ axis according to the following transformation

$x^{\prime}=x \cos \theta^{\prime}+y \sin \theta^{\prime}$

$y^{\prime}=-x \sin \theta^{\prime}+y \cos \theta^{\prime}$

$z^{\prime}=z$

where $\theta^{\prime}=\pi-\theta_{\text {lon. }}$. A plane fit solution of the form same as equation (13) using the deprojected Cartesian coordinates $\left(x^{\prime}, y^{\prime}, z^{\prime}\right)$ obtained above is applied separately for the disk and bar Cepheids. From the coefficients of the plane fits, we find that the inclinations and position angle of lines of nodes of the disk and bar are $\left(25^{\circ} .724 \pm 0^{\circ} .392,148^{\circ} .060 \pm 1^{\circ} .444\right)$ and $\left(46^{\circ} .169 \pm 3^{\circ} .652,113^{\circ} .303 \pm 4^{\circ} .882\right)$, respectively, with an offset of $1.182 \pm 0.082 \mathrm{kpc}$ between the two, the bar being closer to us by $\sim 1 \mathrm{kpc}$ from the disk of the LMC. These results are in good agreement with the postulates put forwarded by Zhao \& Evans (2000) in order to explain the microlensing optical depth observed towards the LMC. In the model proposed by Zhao \& Evans (2000), it was postulated that the disk and bar stars of the LMC are slightly misaligned with different inclinations having an offset of $\sim 1 \mathrm{kpc}$ along the line of sight. Based on relative distance measurements of more than 2000 Cepheids, Nikolaev et al. (2004) 
found that the bar of the LMC is located $\sim 0.5 \mathrm{kpc}$ in front of the main LMC disk, where it was suggested as a lower limit on the actual offset. Using the magnitudes of Red Clump stars, Koerwer (2009) also found the bar to be floating over the disk by $\sim 1 \mathrm{kpc}$ and is nearer to us. van der Marel \& Kallivayalil (2014) also obtained the dynamical center of the rotating HI disk of the LMC to be $\geq 1$ kpc away from the densest point in the bar. These findings are in excellent agreement with the results obtained in the present study. The existence of an offset between the disk and bar of the LMC is further substantiated using the two sample Kolmogorov-Smirnov (KS) statistics. The KS test allows to reject or confirm the hypothesis whether the two samples (data sets) have a common parent distribution. The two sample KS test returns a distance statistics $D$ defined as the maximum distance between the cumulative distribution functions (CDFs) of the two samples along with the corresponding probability $P(D)$ if the two samples are drawn from the same parent distribution or not. A probability with $P(D)<10 \%$ indicates that the two distributions are significantly different (Press et al. 2002). CDFs of line of sight distances of disk (sample 1) and bar (sample 2) Cepheids are shown in Fig. 11. In order to check whether the two samples came from the same distribution at a significance level of 0.001 we use the two sample KS statistics. The KS test applied on the distance CDFs yields $D$-value $=0.512$ with $P$-value $=0.000$. These values indicate the CDFs of the two samples are different at a significance level of 0.001 which implies that the LMC bar region is closer to us than its disk. Not only there exists differences in the structural parameters between the LMC bar and disk as found in the present study, but also subtle differences in the $[\alpha / F e]$ values between these two components of the LMC were confirmed by Van der Swaelmen et al. (2013). These findings reflect the fact that bar is not merely an overdensity but related to a fresh episode of star formation in the central parts of the LMC around $\sim 5$ Gyr ago while the star formation history of the bar and inner disk were similar at earlier epochs (Van der Swaelmen et al. 2013).

After finding that the bar of the LMC is nearer to us than the entire disk of the LMC, we now compare the CDFs between (i) the bar and the northern part of the LMC disk ( $y>0$ and excluding the bar region) (ii) northern part and southwestern part of the LMC disk $(y<0$ and excluding the bar region). The CDFs of the comparison between these two different regions are shown in Fig. 12. The KS test performed on these CDFs show that the bar of the LMC lies at a closer distance than the northern part of the disk whereas, the southwestern parts of the LMC disk are located at the farthest distance of the LMC from the line of sight of a terrestrial observer. Similar test performed on the western and southern parts of the LMC disk indicates that southern part is situated more nearer to us than its western part.

\section{SUMMARY AND CONCLUSIONS}

In this paper, we have utilised the mutli-wavelength archival data of more than 3500 common classical Cepheids available in seven photometric bands, viz., $V, I, J, H, K s,[3.6] \mu m$ and [4.5] $\mu \mathrm{m}$ to determine the geometrical and viewing angle pa-

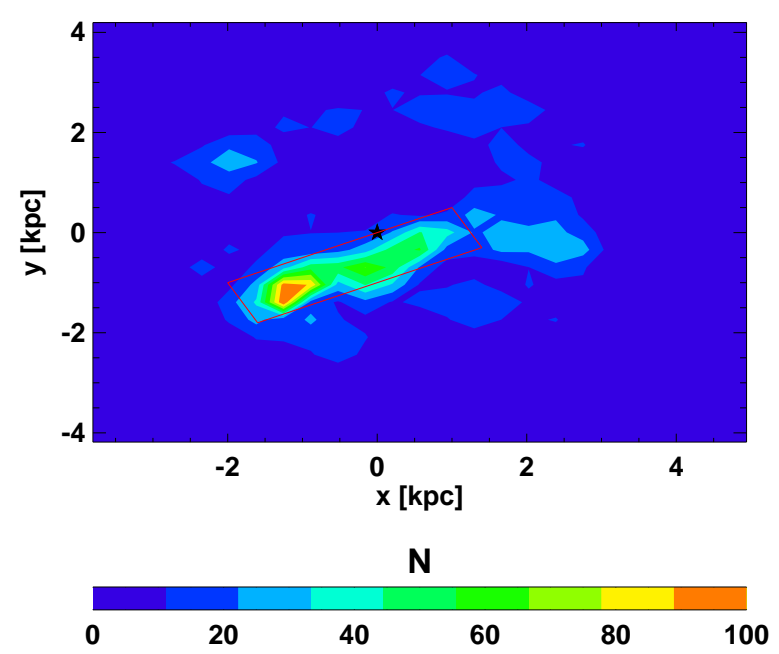

Figure 10. Two dimensional density contours of the LMC. The bar is shown as the rectangular region in the $X Y$-plane satisfying the inequality given by equation (14).

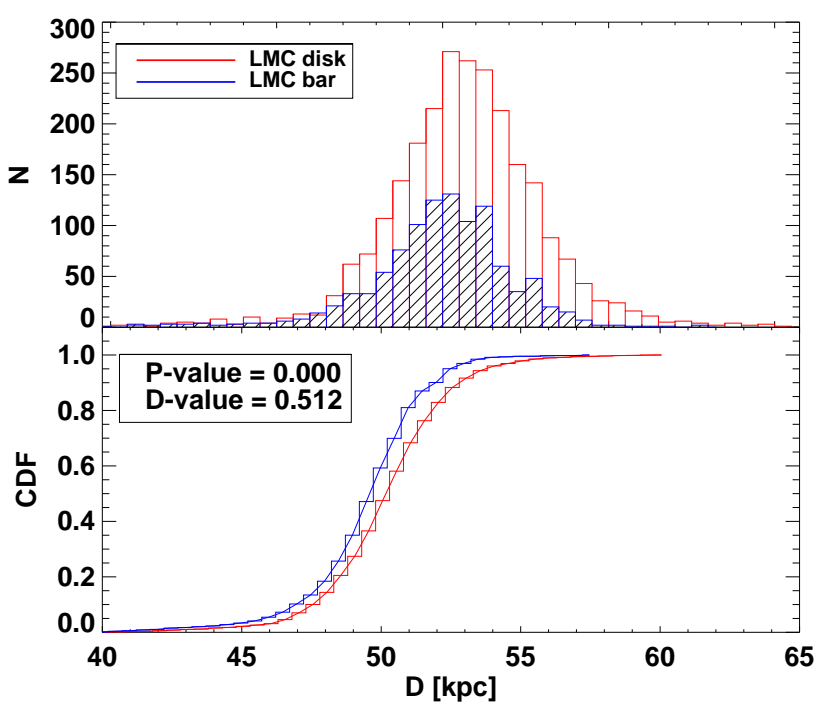

Figure 11. Cumulative distributions of line of sight distances of disk and bar Cepheids are shown as red and blue solid lines, respectively. The bar and disk Cepheids are selected whether the inequalities defined in equation (14) are satisfied or not.

rameters of the LMC. Solving the PL relations simultaneously in these bands, we obtain the following results:

(i) Relative values of reddening and true distance moduli for individual classical Cepheids with respect to the mean values of reddening and distance modulus of the LMC. In order to determine these values, apparent distance moduli calculated using the mean magnitudes and the PL relations 

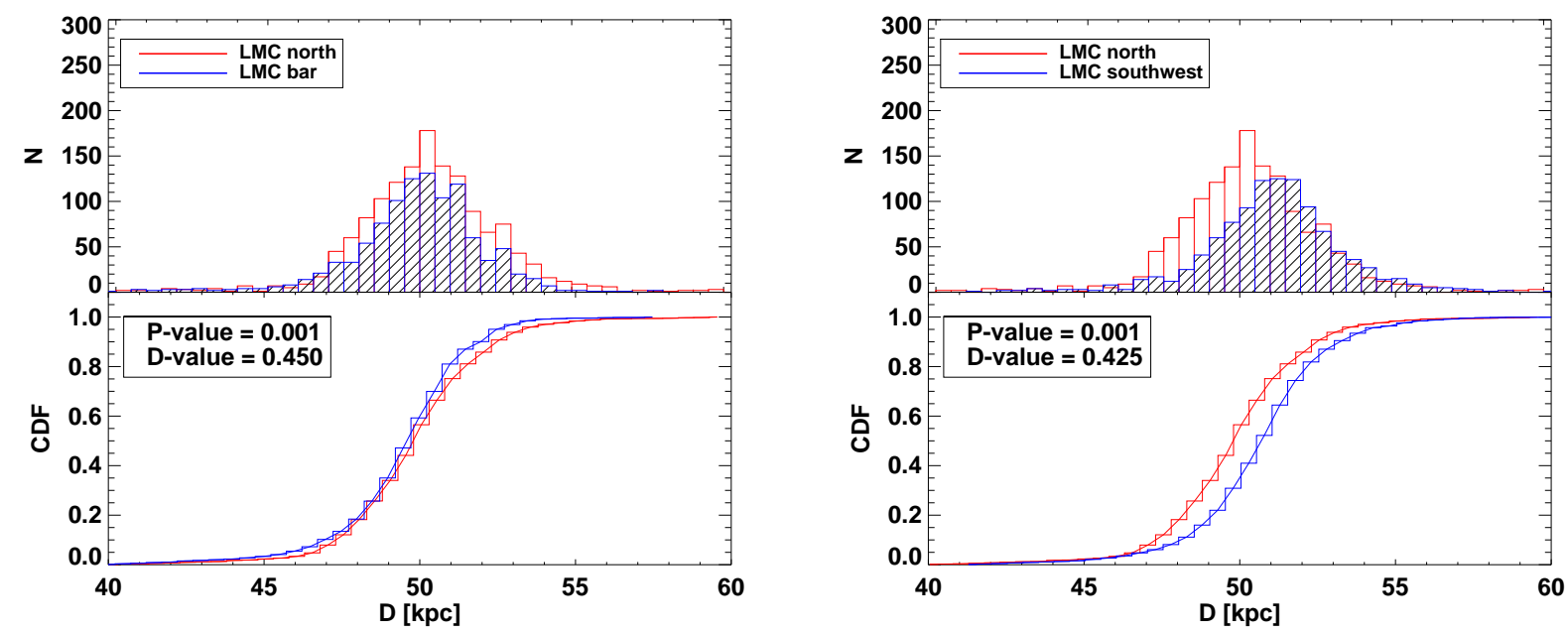

Figure 12. CDFs of line of sight distances between the bar and the northern part of the LMC disk as well as between the northern and the southwestern parts of the LMC disk are shown in the left and right panels, respectively.

obtained in each of the seven photometric bands are fitted simultaneously as a function of inverse wavelength $(1 / \lambda)$ using the Cardelli et al. (1989) reddening law.

(ii) The PL relations are obtained in two iterations. In iteration 1, these relations are obtained without any reddening and distance corrections. In iteration 2 , the PL relations are obtained by correcting the relative distance and reddening values obtained using the relations found in iteration 1. The dispersions in the PL relations obtained from iteration 2 decrease substantially due to the distance and reddening corrections leading to the much more improved relations. The small residual dispersions in the improved PL relations exist due to the intrinsic error accounting for the finite width of the instability strip as well as photometric errors corresponding to different photometric bands. Since the amplitudes of the light curves of Cepheids are a function of wavelengths and decrease while going from optical to midinfrared photometric bands, the photometric errors increase accordingly. This is reflected in the resulting PL relations as we go from optical to mid-infrared photometric bands.

(iii) The reddening and distance modulus offsets obtained from the simultaneous fitting of apparent distance moduli in seven photometric bands are converted into their absolute values of reddening and true distance modulus using the mean values of reddening and distance modulus of the LMC taken from the literature.

(iv) The reddening map of the LMC is constructed from the absolute reddening values distributed in the $(\alpha, \delta)$ coordinates. Two prominent regions having higher values of reddening can be identified from the map. One of them is the 30 Dor region which has the highest reddening values. The other one with higher values of reddening can be associated with the LMC HI supergiant shells SGS (LMC). The reddening map constructed in this paper is found to be in excellent agreement with those found by Nikolaev et al. (2004) and Inno et al. (2016), respectively.

(v) The values of distance along with the information of equatorial coordinates $(\alpha, \delta)$ are used to convert them into the corresponding Cartesian coordinates with respect to the plane of the sky. By fitting a plane solution of the form $z=f(x, y)$ to the observed three dimensional distribution of the LMC Cepheids, we find the following viewing angle parameters: inclination angle $i=25^{\circ} .110 \pm 0^{\circ} .365$ and position angle of line of nodes $\theta_{\text {lon }}=154^{\circ} .702 \pm 1^{\circ} .378$. On the other hand, modelling the observed three dimensional distribution of the Cepheids as a triaxial ellipsoid, the following values of the geometrical axes ratios of the LMC are obtained: $1.000 \pm 0.003: 1.151 \pm 0.003: 1.890 \pm 0.014$ with the viewing angle parameters: inclination angle of $i=11^{\circ} .920 \pm 0^{\circ} .315$ with respect to the longest axis from the line of sight and position angle of line of nodes $\theta_{\text {lon }}=128^{\circ} .871 \pm 0^{\circ} .569$ as measured eastwards from north following astronomical convention.

(vi) Separating the bar and disk of the LMC, we find that the bar and disk have significantly different values of position angles of line of nodes as well as inclinations, i.e. they are misaligned with respect to each other. An offset of $\sim 1$ kpc has also been obtained between the two with the bar being closer to us than the disk. Higher value of inclination angle found for the bar also corroborates the fact that it is closer to us than the disk. These values obtained from the precise multi-wavelength observational data of classical Cepheids provide evidences in support of the postulates of the model proposed by Zhao \& Evans (2000) as an explanation of the microlensing optical depth observed towards the LMC.

\section{ACKNOWLEDGMENTS}

The authors acknowledge the use of highly valuable publicly available OGLE-IV data for this study. SD thanks Science and Engineering Research Board (SERB), Department of Science \& Technology (DST), Govt. of India for financial support of this study through a research grant D.O No. SB/FTP/PS-029/2013 under the Fast Track Scheme for Young Scientists in Physical Sciences. CCN thanks the funding from Ministry of Science and Technology (Taiwan) under the contract 104-2112-M-008-012-MY3. HPS and SMK ac- 
knowledge support from Indo-US Science \& Technology Forum (IUSSTF) through the Indo-US virtual networked center on "Theoretical Analyses of Variable Star Data". Lastly, the authors thank the anonymous referee for making various helpful comments and useful suggestions which made the paper significantly relevant. The paper makes use of the facility from arxiv.org/archive/astro-ph, NASA ADS and SIMBAD databases.

\section{REFERENCES}

Bekki K., 2009, MNRAS, 393, L60

Besla G., Kallivayalil N., Hernquist L., van der Marel R. P., Cox T. J., Kereš D., 2012, MNRAS, 421, 2109

Bhardwaj A., Kanbur S. M., Macri L. M., Singh H. P., Ngeow C.-C., Wagner-Kaiser R., Sarajedini A., 2016a, AJ, 151, 88

Bhardwaj A., Ngeow C.-C., Kanbur S. M., Singh H. P., 2016b, MNRAS, 458, 3705

Bhardwaj A., Macri L. M., Rejkuba M., Kanbur S. M., Ngeow C.-C., Singh H. P., 2017, AJ, 153, 154

Bonanos A. Z., et al., 2010, AJ, 140, 416

Cardelli J. A., Clayton G. C., Mathis J. S., 1989, ApJ, 345, 245

Deb S., Singh H. P., 2014, MNRAS, 438, 2440

Deb S., Singh H. P., Kumar S., Kanbur S. M., 2015, MNRAS, 449, 2768

Evans C. J., et al., 2011, A\&A, 530, A108

Freedman W. L., 1988, ApJ, 326, 691

Freedman W. L., Madore B. F., 1990, ApJ, 365, 186

Freedman W. L., et al., 2001, ApJ, 553, 47

Freedman W. L., Madore B. F., Rigby J., Persson S. E., Sturch L., 2008, ApJ, 679, 71

Freedman W. L., et al., 2011, AJ, 142, 192

Glatt K., Grebel E. K., Koch A., 2010, A\&A, 517, A50

Haschke R., Grebel E. K., Duffau S., 2011, AJ, 141, 158

Inno L., et al., 2015, A\&A, 576, A30

Inno L., et al., 2016, ApJ, 832, 176

Jacyszyn-Dobrzeniecka A. M., et al., 2016, Acta Astron., 66, 149

Koerwer J. F., 2009, AJ, 138, 1

Kruk S. J., et al., 2017, MNRAS, 469, 3363

Leavitt H. S., 1908, Annals of Harvard College Observatory, 60, 87

Leavitt H. S., Pickering E. C., 1912, Harvard College Observatory Circular, 173, 1

Mancini L., Calchi Novati S., Jetzer P., Scarpetta G., 2004, A\&A, 427,61

Meixner M., et al., 2006, AJ, 132, 2268

Neilson H. R., Ngeow C.-C., Kanbur S. M., Lester J. B., 2010, ApJ, 716, 1136

Ngeow C., Kanbur S. M., 2008, ApJ, 679, 76

Ngeow C.-C., Kanbur S. M., Neilson H. R., Nanthakumar A., Buonaccorsi J., 2009a, ApJ, 693, 691

Ngeow C., Kanbur S., Ghobrial L., Neilson H., Macri L., 2009b, in Guzik J. A., Bradley P. A., eds, American Institute of Physics Conference Series Vol. 1170, American Institute of Physics Conference Series. pp 37-39, doi:10.1063/1.3246519

Ngeow C.-C., Ita Y., Kanbur S. M., Neilson H., Onaka T., Kato D., 2010, MNRAS, 408, 983

Nikolaev S., Drake A. J., Keller S. C., Cook K. H., Dalal N., Griest K., Welch D. L., Kanbur S. M., 2004, ApJ, 601, 260

Pardy S. A., D'Onghia E., Athanassoula E., Wilcots E. M., Sheth K., 2016, ApJ, 827, 149

Paz D. J., Lambas D. G., Padilla N., Merchán M., 2006, MNRAS, 366,1503

Pejcha O., Stanek K. Z., 2009, ApJ, 704, 1730

Persson S. E., Madore B. F., Krzemiński W., Freedman W. L., Roth M., Murphy D. C., 2004, AJ, 128, 2239

Pietrzyński G., et al., 2013, Nature, 495, 76
Press W. H., Teukolsky S. A., Vetterling W. T., Flannery B. P., 2002, Numerical recipes in $\mathrm{C}++$ : the art of scientific computing

Rich J. A., Persson S. E., Freedman W. L., Madore B. F., Monson A. J., Scowcroft V., Seibert M., 2014, ApJ, 794, 107

Scowcroft V., Seibert M., Freedman W. L., Beaton R. L., Madore B. F., Monson A. J., Rich J. A., Rigby J. R., 2016, MNRAS, 459,1170

Soszyński I., et al., 2015, Acta Astron., 65, 297

Subramaniam A., 2005, A\&A, 430, 421

Subramanian S., Subramaniam A., 2012, ApJ, 744, 128

Subramanian S., Subramaniam A., 2013, A\&A, 552, A144

Tatton B. L., et al., 2013, A\&A, 554, A33

Van der Swaelmen M., Hill V., Primas F., Cole A. A., 2013, A\&A, $560, \mathrm{~A} 44$

Westerlund B. E., 1997, Cambridge Astrophysics Series, 29

Yozin C., Bekki K., 2014, MNRAS, 439, 1948

Zaritsky D., 1999, AJ, 118, 2824

Zaritsky D., Harris J., Thompson I. B., Grebel E. K., 2004, AJ, 128, 1606

Zhao H., Evans N. W., 2000, ApJ, 545, L35

de Vaucouleurs G., Freeman K. C., 1972, Vistas in Astronomy, 14,163

de Vaucouleurs G., de Vaucouleurs A., Corwin Jr. H. G., Buta R. J., Paturel G., Fouque P., 1991, Sky \& Telesc., 82, 621

van der Marel R. P., Cioni M.-R. L., 2001, AJ, 122, 1807

van der Marel R. P., Kallivayalil N., 2014, ApJ, 781, 121

This paper has been typeset from a $\mathrm{T}_{\mathrm{E}} \mathrm{X} / \mathrm{LAT}_{\mathrm{E}} \mathrm{X}$ file prepared by the author. 